Ref. SISSA 28/2005/EP

CERN-PH-TH/2005-082

May 2005

hep-ph/0505226

\title{
The Absolute Neutrino Mass Scale, Neutrino Mass Spectrum, Majorana CP-Violation and Neutrinoless Double-Beta Decay
}

\author{
S. Pascoli ${ }^{a)}$, S. T. Petcov ${ }^{b, c)} 1$ and T. Schwetz ${ }^{b)}$ \\ a) Theory Division, CERN, CH-1211 Geneva 23, Switzerland \\ b) Scuola Internazionale Superiore di Studi Avanzati, I-34014 Trieste, Italy \\ ${ }^{c}$ Istituto Nazionale di Fisica Nucleare, Sezione di Trieste, I-34014 Trieste, Italy
}

\begin{abstract}
Assuming 3- $\nu$ mixing, massive Majorana neutrinos and neutrinoless double-beta $\left((\beta \beta)_{0 \nu^{-}}\right)$decay generated only by the $(V-A)$ charged current weak interaction via the exchange of the three Majorana neutrinos, we briefly review the predictions for the effective Majorana mass $|<m>|$ in $(\beta \beta)_{0 \nu}$-decay and reanalyse the physics potential of future $(\beta \beta)_{0 \nu}$-decay experiments to provide information on the type of neutrino mass spectrum, the absolute scale of neutrino masses, and Majorana CP-violation in the lepton sector. Using as input the most recent experimental results on neutrino oscillation parameters and the prospective precision that can be achieved in future measurements of the latter, we perform a statistical analysis of a $(\beta \beta)_{0 \nu}$-decay half-life measurement taking into account experimental and theoretical errors, as well as the uncertainty implied by the imprecise knowledge of the corresponding nuclear matrix element (NME). We show, in particular, how the possibility to discriminate between the different types of neutrino mass spectra and the constraints on the absolute neutrino mass scale depend on the mean value and the experimental error of $|\langle m\rangle|$ and on the NME uncertainty. The constraints on Majorana CP-violation phases in the neutrino mixing matrix, which can be obtained from a measurement of $|\langle m\rangle|$ and additional data on the sum of neutrino masses, are also investigated in detail. We estimate the required experimental accuracies on both types of measurements, and the required precision in the NME permitting to address the issue of Majorana CP-violation in the lepton sector.
\end{abstract}

\footnotetext{
${ }^{1}$ Also at: Institute of Nuclear Research and Nuclear Energy, Bulgarian Academy of Sciences, 1784 Sofia, Bulgaria
} 


\section{Introduction}

There has been remarkable progress in the studies of neutrino oscillations in the last several years. The experiments with solar, atmospheric, reactor and accelerator neutrinos [1-7] have provided compelling evidence for the existence of neutrino oscillations caused by nonzero neutrino masses and neutrino mixing ${ }^{1}$. The latest addition to these results are the Super-Kamiokande (SK) data on the $L / E$-dependence of the (essentially multi-GeV) $\mu$-like atmospheric neutrino events [9], $L$ and $E$ being the distance traveled by neutrinos and the neutrino energy, and the new spectrum data of the KamLAND (KL) and K2K experiments $[10,11]$. For the first time the data directly exhibit the effects of the oscillatory dependence on $L / E$ and $E$ of the probabilities of $\nu$-oscillations in vacuum [12]. As a result of these magnificent developments, the oscillations of solar $\nu_{e}$, atmospheric $\nu_{\mu}$ and $\bar{\nu}_{\mu}$, accelerator $\nu_{\mu}$ (at $L \sim 250 \mathrm{~km}$ ) and reactor $\bar{\nu}_{e}$ (at $L \sim 180 \mathrm{~km}$ ), driven by non-zero $\nu$-masses and $\nu$-mixing, can be considered as practically established.

The evidences for $\nu$-oscillations obtained in the solar and atmospheric neutrino and KL and $\mathrm{K} 2 \mathrm{~K}$ experiments imply the existence of $3-\nu$ mixing in the weak charged-lepton current:

$$
\nu_{l \mathrm{~L}}=\sum_{j=1}^{3} U_{l j} \nu_{j \mathrm{~L}}, \quad l=e, \mu, \tau,
$$

where $\nu_{l L}$ are the flavour neutrino fields, $\nu_{j \mathrm{~L}}$ is the field of neutrino $\nu_{j}$ having a mass $m_{j}$ and $U$ is the Pontecorvo-Maki-Nakagawa-Sakata (PMNS) mixing matrix [13], $U \equiv U_{\text {PMNS }}$. All existing $\nu$-oscillation data, except the data of the LSND experiment [8], can be described assuming 3- $\nu$ mixing in vacuum; we will consider this possibility in what follows ${ }^{2}$.

The PMNS matrix can be parametrized by 3 angles, and, depending on whether the massive neutrinos $\nu_{j}$ are Dirac or Majorana particles, by 1 or $3 \mathrm{CP}$-violation (CPV) phases $[17,18]$. In the standardly used parametrization (see, e.g. [19]), $U_{\mathrm{PMNS}}$ has the form:

$$
U_{\mathrm{PMNS}}=\left(\begin{array}{ccc}
c_{12} c_{13} & s_{12} c_{13} & s_{13} \\
-s_{12} c_{23}-c_{12} s_{23} s_{13} e^{i \delta} & c_{12} c_{23}-s_{12} s_{23} s_{13} e^{i \delta} & s_{23} c_{13} e^{i \delta} \\
s_{12} s_{23}-c_{12} c_{23} s_{13} e^{i \delta} & -c_{12} s_{23}-s_{12} c_{23} s_{13} e^{i \delta} & c_{23} c_{13} e^{i \delta}
\end{array}\right) \operatorname{diag}\left(1, e^{i \frac{\alpha_{21}}{2}}, e^{i \frac{\alpha_{31}}{2}}\right),
$$

where $c_{i j}=\cos \theta_{i j}, s_{i j}=\sin \theta_{i j}$, the angles $\theta_{i j}=[0, \pi / 2], \delta=[0,2 \pi]$ is the Dirac CPV phase and $\alpha_{21}, \alpha_{31}$ are two Majorana CPV phases [17,18]. One can identify the neutrino mass squared difference responsible for solar neutrino oscillations, $\Delta m_{\odot}^{2}$, with $\Delta m_{21}^{2} \equiv m_{2}^{2}-m_{1}^{2}$, $\Delta m_{\odot}^{2}=\Delta m_{21}^{2}>0$. The neutrino mass squared difference driving the dominant $\nu_{\mu} \rightarrow \nu_{\tau}$ $\left(\bar{\nu}_{\mu} \rightarrow \bar{\nu}_{\tau}\right)$ oscillations of atmospheric $\nu_{\mu}\left(\bar{\nu}_{\mu}\right)$ is then given by $\left|\Delta m_{\mathrm{A}}^{2}\right|=\left|\Delta m_{31}^{2}\right| \cong\left|\Delta m_{32}^{2}\right| \gg$ $\Delta m_{21}^{2}$. The corresponding solar and atmospheric neutrino mixing angles, $\theta_{\odot}$ and $\theta_{\mathrm{A}}$, coincide with $\theta_{12}$ and $\theta_{23}$, respectively. The angle $\theta_{13}$ is limited by the data from the CHOOZ and Palo Verde experiments [20].

\footnotetext{
${ }^{1}$ Indications for $\nu$-oscillations were reported also by the LSND collaboration [8].

${ }^{2}$ The interpretation of LSND data in terms of $\bar{\nu}_{\mu} \rightarrow \bar{\nu}_{e}$ oscillations requires $\left(\Delta m^{2}\right)_{\mathrm{LSND}} \simeq 1 \mathrm{eV}^{2}$. The minimal 4-neutrino mixing scheme, which could incorporate the LSND indications for $\bar{\nu}_{\mu} \rightarrow \bar{\nu}_{e}$ oscillations, is strongly disfavored by the data [14]. The $\nu$-oscillation explanation of the LSND results is possible, assuming 5-neutrino mixing [15]. The LSND results are being tested in the MiniBooNE experiment [16].
} 
Thus, the basic phenomenological parameters characterising the $3-\nu$ mixing are: i) the 3 angles $\theta_{12}, \theta_{23}, \theta_{13}$, ii) depending on the nature of massive neutrinos 1 Dirac $(\delta)$ or 1 Dirac +2 Majorana $\left(\delta, \alpha_{21}, \alpha_{31}\right) \mathrm{CPV}$ phases, and iii) the 3 neutrino masses, $m_{1}, m_{2}, m_{3}$. Getting precise information about the $\nu$-mixing parameters is of fundamental importance for understanding the origin of neutrino mixing (see, e.g. [21]).

The existing neutrino oscillation data allow us to determine $\Delta m_{\odot}^{2},\left|\Delta m_{\mathrm{A}}^{2}\right|, \sin ^{2} \theta_{\odot}$ and $\sin ^{2} 2 \theta_{\mathrm{A}}$ with a relatively good precision and to obtain rather stringent limits on $\sin ^{2} \theta_{13}$ (see, e.g. $[5,10,14,22,23]$ ). The data imply that $\Delta m_{\odot}^{2}=\Delta m_{21}^{2} \sim 8.0 \times 10^{-5} \mathrm{eV}^{2},\left|\Delta m_{\mathrm{A}}^{2}\right| \sim$ $2.2 \times 10^{-3} \mathrm{eV}^{2}, \sin ^{2} \theta_{\odot} \sim 0.30, \sin ^{2} 2 \theta_{\mathrm{A}} \sim 1$ and $\sin ^{2} \theta_{13}<0.05$. The sign of $\Delta m_{\mathrm{A}}^{2}$, as is well known, cannot be determined from the present (SK atmospheric neutrino and K2K) data. In the case of 3- $\nu$ mixing the two possibilities, $\Delta m_{31(32)}^{2}>0$ or $\Delta m_{31(32)}^{2}<0$ correspond to two different types of $\nu$-mass spectrum:

- with normal hierarchy (or ordering), $m_{1}<m_{2}<m_{3}, \Delta m_{\mathrm{A}}^{2}=\Delta m_{31}^{2}>0$, and - with inverted hierarchy (ordering) ${ }^{3}, m_{3}<m_{1}<m_{2}, \Delta m_{\mathrm{A}}^{2}=\Delta m_{32}^{2}<0$.

Depending on the sign of $\Delta m_{\mathrm{A}}^{2}, \operatorname{sgn}\left(\Delta m_{\mathrm{A}}^{2}\right)$, and the value of the lightest neutrino mass, $\min \left(m_{j}\right)$, the $\nu$-mass spectrum can be

- Normal Hierarchical (NH):

$m_{1} \ll m_{2} \ll m_{3}$, with $m_{2} \cong \sqrt{\Delta m_{\odot}^{2}} \sim 0.009 \mathrm{eV}$ and $m_{3} \cong \sqrt{\left|\Delta m_{\mathrm{A}}^{2}\right|} \sim 0.047 \mathrm{eV}$;

- Inverted Hierarchical (IH):

$m_{3} \ll m_{1}<m_{2}$, with $m_{1,2} \cong \sqrt{\left|\Delta m_{\mathrm{A}}^{2}\right|} \sim 0.047 \mathrm{eV}$;

- Quasi-Degenerate (QD): $m_{1} \cong m_{2} \cong m_{3}$, with $m_{1} \cong m_{2} \cong m_{3} \cong m_{0}, m_{j}^{2} \gg\left|\Delta m_{\mathrm{A}}^{2}\right|, m_{0} \gtrsim 0.10 \mathrm{eV}$.

The precision on the mixing angles $\theta_{21}, \theta_{23}, \theta_{13}$, and on $\Delta m_{21}^{2}$ and $\left|\Delta m_{31}^{2}\right|$, can be significantly improved in future $\nu$-oscillation experiments (see, e.g. [26-30]). The sign of $\Delta m_{31}^{2}$ can be determined by studying oscillations of neutrinos and antineutrinos, say, $\nu_{\mu} \rightarrow \nu_{e}$ and $\bar{\nu}_{\mu} \rightarrow \bar{\nu}_{e}$, in which matter effects are sufficiently large. This can be done in long-baseline $\nu$-oscillation experiments running both with neutrino and antineutrino beams (see, e.g. [31]) or in the neutrino mode only [32,33]. If $\sin ^{2} 2 \theta_{13} \gtrsim 0.05$ and $\sin ^{2} \theta_{23} \gtrsim 0.50$, information on $\operatorname{sgn}\left(\Delta m_{31}^{2}\right)$ might be obtained in atmospheric neutrino experiments by investigating the effects of the subdominant transitions $\nu_{\mu(e)} \rightarrow \nu_{e(\mu)}$ and $\bar{\nu}_{\mu(e)} \rightarrow \bar{\nu}_{e(\mu)}$ of atmospheric neutrinos that traverse the Earth $[34,35]$.

The neutrino oscillation experiments, however, cannot provide information on the absolute scale of neutrino masses (or on $\min \left(m_{j}\right)$ ) and thus on the possible hierarchical structure (NH, IH, QD, etc.) of the neutrino mass spectrum. The oscillations of flavour neutrinos, $\nu_{l} \rightarrow \nu_{l^{\prime}}$ and $\bar{\nu}_{l} \rightarrow \bar{\nu}_{l^{\prime}}, l, l^{\prime}=e, \mu, \tau$, are insensitive to the nature-Dirac or Majorana-of massive neutrinos $\nu_{j}$; they are insensitive to the Majorana CPV phases $\alpha_{21,31}[17,36]$. If $\nu_{j}$ are Majorana fermions, getting experimental information about the Majorana CPV phases

\footnotetext{
${ }^{3}$ In the convention we use (called A), the neutrino masses are not ordered in magnitude according to their index number: $\Delta m_{31}^{2}<0$ corresponds to $m_{3}<m_{1}<m_{2}$. We can also always number the neutrinos with definite mass, in such a way that [24] $m_{1}<m_{2}<m_{3}$. In this convention (called B) we have in the case of the inverted hierarchy spectrum: $\Delta m_{\odot}^{2}=\Delta m_{32}^{2}, \Delta m_{\mathrm{A}}^{2}=\Delta m_{31}^{2}$. Convention B is used, e.g. in [19,25].
} 
in $U_{\text {PMNS }}$ would be a remarkably challenging problem ${ }^{4}[24,39-41]$.

Establishing whether $\nu_{j}$ are Dirac fermions possessing distinct antiparticles, or are Majorana fermions, i.e. spin $1 / 2$ particles that are identical with their antiparticles, is of fundamental importance for understanding the underlying symmetries of particle interactions and the origin of $\nu$-masses. Let us recall that neutrinos $\nu_{j}$ with definite mass will be Dirac fermions if particle interactions conserve some additive lepton number, e.g. the total lepton charge $L=L_{e}+L_{\mu}+L_{\tau}$. If no lepton charge is conserved, the neutrinos $\nu_{j}$ will be Majorana fermions (see, e.g. [42]). The observed patterns of $\nu$-mixing and of $\left|\Delta m_{\mathrm{A}}^{2}\right|$ and $\Delta m_{\odot}^{2}$ can be related to Majorana $\nu_{j}$ and the existence of an approximate symmetry corresponding to the conservation of the lepton charge $L^{\prime}=L_{e}-L_{\mu}-L_{\tau}[43,44]$. The massive neutrinos are predicted to be of Majorana nature by the see-saw mechanism of neutrino mass generation [45], which also provides an attractive explanation of the smallness of neutrino masses and, through the leptogenesis theory [38], of the observed baryon asymmetry of the Universe. Determining the nature (Dirac or Majorana) of massive neutrinos $\nu_{j}$ is one of the fundamental problems in the studies of neutrino mixing (see, e.g. [21]).

If neutrinos $\nu_{j}$ are Majorana fermions, processes in which the total lepton charge $L$ is not conserved and changes by two units, such as $K^{+} \rightarrow \pi^{-}+\mu^{+}+\mu^{+}, \mu^{+}+(A, Z) \rightarrow$ $(A, Z+2)+\mu^{-}$, etc., should exist ${ }^{5}$. The only feasible experiments that at present have the potential of establishing the Majorana nature of massive neutrinos are the experiments searching for the neutrinoless double beta $\left((\beta \beta)_{0 \nu}\right)$-decay $(A, Z) \rightarrow(A, Z+2)+e^{-}+e^{-}$(see, e.g. $[42,47,48])$. Under the assumption of $(\beta \beta)_{0 \nu}$-decay generated only by the $(V$ - $A)$ charged current weak interaction via the exchange of the three Majorana neutrinos $\nu_{j}\left(m_{j} \lesssim 1 \mathrm{eV}\right)$, the dependence of the $(\beta \beta)_{0 \nu}$-decay amplitude $A(\beta \beta)_{0 \nu}$ on the neutrino mass and mixing parameters factorizes in the effective Majorana mass $<m>$ (see, e.g. $[42,49])$ :

$$
A(\beta \beta)_{0 \nu} \sim<m>\mathcal{M}
$$

where $\mathcal{M}$ is the corresponding nuclear matrix element (NME) and $|<m>|$ is given by

$$
|<m>|=\left.\left|m_{1}\right| U_{\mathrm{e} 1}\right|^{2}+m_{2}\left|U_{\mathrm{e} 2}\right|^{2} e^{i \alpha_{21}}+m_{3}\left|U_{\mathrm{e} 3}\right|^{2} e^{i \alpha_{31}} \mid \text {. }
$$

If CP-invariance holds ${ }^{6}$, one has [50] $\alpha_{21}=k \pi, \alpha_{31}=k^{\prime} \pi$, where $k, k^{\prime}=0,1,2, \ldots$, and

$$
\eta_{21} \equiv e^{i \alpha_{21}}= \pm 1, \quad \eta_{31} \equiv e^{i \alpha_{31}}= \pm 1
$$

represent the relative CP-parities of Majorana neutrinos $\nu_{1}$ and $\nu_{2}$, and $\nu_{1}$ and $\nu_{3}$, respectively. As eq. (3) indicates, the observation of $(\beta \beta)_{0 \nu}$-decay of a given nucleus, and the measurement of the corresponding half-life, would allow a determination of $|\langle m\rangle|$ only if the value of the relevant NME is known with a relatively small uncertainty.

The experimental searches for $(\beta \beta)_{0 \nu}$-decay have a long history (see, e.g. [47, 49]). The best sensitivity was achieved in the Heidelberg-Moscow ${ }^{76}$ Ge experiment [51]:

$$
|<m>|<(0.35-1.05) \mathrm{eV}, \quad \text { at } 90 \% \text { C.L. }
$$

\footnotetext{
${ }^{4}$ The phases $\alpha_{21,31}$ can significantly affect the predictions for the rates of (LFV) decays $\mu \rightarrow e+\gamma$, $\tau \rightarrow \mu+\gamma$, etc. in a large class of supersymmetric theories with see-saw mechanism of $\nu$-mass generation (see, e.g. [37]). Majorana CPV phases might be at the origin of baryon asymmetry of the Universe [38].

${ }^{5}$ The existing experimental constraints on the $|\Delta L|=2$ processes have been discussed recently in, e.g. [46].

${ }^{6}$ We assume that $m_{j}>0$ and that the fields of the Majorana neutrinos $\nu_{j}$ satisfy the Majorana condition: $C\left(\bar{\nu}_{j}\right)^{T}=\nu_{j}, j=1,2,3$, where $C$ is the charge conjugation matrix.
} 
where a factor of 3 uncertainty associated with the calculation of the relevant nuclear matrix element [49] is taken into account. A similar result has been obtained by the IGEX collaboration [52]: $|<m>|<(0.33-1.35) \mathrm{eV}$ (90\% C.L.). A positive signal at $>3 \sigma$, corresponding to $|<m>|=(0.1-0.9) \mathrm{eV}$ at $99.73 \%$ C.L., is claimed to be observed in [53]. This result will be checked in the currently running and future $(\beta \beta)_{0 \nu}$-decay experiments. Two experiments, NEMO3 (with ${ }^{100} \mathrm{Mo}$ and ${ }^{82} \mathrm{Se}$ ) [54] and CUORICINO (with ${ }^{130} \mathrm{Te}$ ) [55], designed to reach a sensitivity of $|<m>| \sim(0.2-0.3) \mathrm{eV}$, are taking data. Their first results read (90\% C.L.):

$$
|<m>|<(0.7-1.2) \mathrm{eV}[54], \quad|<m>|<(0.2-1.1) \mathrm{eV} \quad[55]
$$

where the estimated uncertainties in the NME are accounted for. A number of projects aim to reach a sensitivity to $|<m>| \sim(0.01-0.05)$ eV [48]: CUORE $\left({ }^{130} \mathrm{Te}\right)$, GERDA $\left({ }^{76} \mathrm{Ge}\right)$, EXO $\left({ }^{136} \mathrm{Xe}\right)$, MAJORANA $\left({ }^{76} \mathrm{Ge}\right)$, MOON $\left({ }^{100} \mathrm{Mo}\right)$, XMASS $\left({ }^{136} \mathrm{Xe}\right)$, CANDLES $\left({ }^{48} \mathrm{Ca}\right)$, SuperNEMO, etc. These experiments, in particular, can test the positive result claimed in [53] and probe the region of values of $|\langle m\rangle|$ predicted in the case of IH and QD spectra [25].

In the present article we reanalyze the potential contribution that the future planned $(\beta \beta)_{0 \nu}$-decay experiments can make to the studies of neutrino mixing. The observation of $(\beta \beta)_{0 \nu}$-decay and the measurement of the corresponding half-life with a sufficient accuracy, would not only be a proof that the total lepton charge is not conserved in nature (see, e.g. [56]), but might provide also unique information i) on the type and possible hierarchical structure (NH, IH, QD, etc.) of the neutrino mass spectrum [19,25], ii) on the absolute scale of neutrino masses [58], and iii) on the Majorana CP-violation phases [24]. We consider $3-\nu$ mixing, assume massive Majorana neutrinos and $(\beta \beta)_{0 \nu}$-decay generated only by the $(V-A)$ charged current weak interaction via the exchange of the three Majorana neutrinos. As input in the analysis we use the results of recent studies of the precision that can be achieved in the measurement of the solar neutrino and CHOOZ mixing angles $\theta_{12}$ and $\theta_{13}$, and of the neutrino mass squared differences $\Delta m_{21}^{2}$ and $\left|\Delta m_{31}^{2}\right|$, on which $|<m>|$ depends. The uncertainty in the measured value of $|<m\rangle \mid$, which is due to the imprecise knowledge of the relevant nuclear matrix elements, is also taken into account. All relevant errors are treated in a statistically self-consistent manner.

Our work is a continuation of earlier studies ${ }^{7}$ (see, e.g. [19,25,41,58-63]). It is stimulated by the remarkable progress recently made in the experimental studies of $\nu$-oscillations $[3,5$, $10,11]$ and by the recent analyses $[26-30,64,65]$ in which the prospects for high precision determination of $\sin ^{2} \theta_{12}, \sin ^{2} \theta_{13}, \Delta m_{21}^{2}$, and $\left|\Delta m_{31}^{2}\right|$ in future $\nu$-oscillation experiments have been extensively investigated. As a result of these studies the experiments that can provide the most precise measurement of the $\nu$-oscillation parameters $|<m\rangle \mid$ depends on, have been identified and a rather thorough evaluation of the precision that can be achieved has been made. In view of these developments a re-examination of the physics potential of the future $(\beta \beta)_{0 \nu}$-decay experiments is both necessary and timely.

The outline of the paper is as follows. In Section 2 we briefly discuss the present status of the determination of, and the prospect for improvements of the precision on, the neutrino oscillation parameters relevant to the analysis of $(\beta \beta)_{0 \nu}$-decay experiments. In Section 3 we review the predictions for the effective Majorana mass $|<m>|$ as a function of the lightest

\footnotetext{
${ }^{7}$ For an extensive list of references see, e.g. [57].
} 
neutrino mass and the type of the neutrino mass spectrum, taking into account present and prospective uncertainties in the neutrino oscillation parameters. In Section 4 we present the results of a quantitative investigation of the potential of a future $(\beta \beta)_{0 \nu}$-decay experiment based on a $\chi^{2}$-analysis. We show our results as a function of quantities such as the observed mean value of $|<m>|$, its experimental uncertainty, and the uncertainty in the NME. We evaluate the possibility to obtain information on the lightest neutrino mass, the type of $\nu$-mass spectrum and Majorana CPV phases from a $(\beta \beta)_{0 \nu}$-decay experiment. In the latter case we take into account the constraint on the sum of neutrino masses $\Sigma$, which could be provided by cosmological observations, and investigate in detail the accuracies on $|<m>|$ and $\Sigma$, required in order to probe Majorana CP-violation in the lepton sector. Finally we conclude in Section 5 .

\section{The Neutrino Mixing Parameters and $|<m>|$}

One can express [66] the two larger neutrino masses in terms of the lightest one, $\min \left(m_{j}\right) \equiv m_{0} \equiv m_{\mathrm{MIN}}$, and of $\Delta m_{\odot}^{2}$ and $\Delta m_{\mathrm{A}}^{2}{ }^{8}$. Within the convention we use, in both cases of $\nu$-mass spectrum with normal and inverted hierarchy, one has: $\Delta m_{\odot}^{2}=\Delta m_{21}^{2}>0$. For normal hierarchy, $\min \left(m_{j}\right)=m_{1}, \Delta m_{\mathrm{A}}^{2}=\Delta m_{31}^{2}>0, m_{2}=\left(m_{1}^{2}+\Delta m_{\odot}^{2}\right)^{\frac{1}{2}}$, and $m_{3}=$ $\left(m_{1}^{2}+\Delta m_{\mathrm{A}}^{2}\right)^{\frac{1}{2}}$. If the spectrum is with inverted hierarchy, $\min \left(m_{j}\right)=m_{3}, \Delta m_{\mathrm{A}}^{2}=\Delta m_{32}^{2}<0$ and thus $m_{1}=\left(m_{3}^{2}+\left|\Delta m_{\mathrm{A}}^{2}\right|-\Delta m_{\odot}^{2}\right)^{\frac{1}{2}} \cong\left(m_{3}^{2}+\left|\Delta m_{\mathrm{A}}^{2}\right|\right)^{\frac{1}{2}}, m_{2}=\left(m_{3}^{2}+\left|\Delta m_{\mathrm{A}}^{2}\right|\right)^{\frac{1}{2}}$. For both types of mass ordering, the following relations hold: $\left|U_{\mathrm{e} 1}\right|^{2}=\cos ^{2} \theta_{\odot}\left(1-\sin ^{2} \theta_{13}\right)$, $\left|U_{\mathrm{e} 2}\right|^{2}=\sin ^{2} \theta_{\odot}\left(1-\sin ^{2} \theta_{13}\right)$, and $\left|U_{\mathrm{e} 3}\right|^{2} \equiv \sin ^{2} \theta_{13}, \theta_{\odot} \equiv \theta_{12}$. Thus, in the case of interest the effective Majorana mass $|<m>|$, eq. (41), depends in general on: i) $\Delta m_{\mathrm{A}}^{2}=\Delta m_{31(32)}^{2}$, ii) $\theta_{\odot}=\theta_{12}$ and $\Delta m_{\odot}^{2}=\Delta m_{21}^{2}$, iii) the lightest neutrino mass $m_{0}$, iv) the mixing angle $\theta_{13}$, and v) the Majorana CPV phases $\alpha_{21,31}$.

The best fit value and the $95 \%$ C.L. allowed range of $\left|\Delta m_{\mathrm{A}}^{2}\right|$ found in a combined analysis of the atmospheric neutrino ${ }^{9}$ and $\mathrm{K} 2 \mathrm{~K}$ data read $[5,14]$ :

$$
\begin{gathered}
\left|\Delta m_{\mathrm{A}}^{2}\right|=2.2 \times 10^{-3} \mathrm{eV}^{2}, \\
\left|\Delta m_{\mathrm{A}}^{2}\right|=(1.7-2.9) \times 10^{-3} \mathrm{eV}^{2} .
\end{gathered}
$$

Combined 2- $\nu$ oscillation analyses of the solar neutrino and KL 766.3 tyr spectrum data show $[10,22]$ that $\Delta m_{\odot}^{2}$ and $\theta_{\odot}$ lie in the low-LMA region: $\Delta m_{\odot}^{2}=\left(7.9_{-0.5}^{+0.6}\right) \times 10^{-5} \mathrm{eV}^{2}$, $\tan ^{2} \theta_{\odot}=\left(0.40_{-0.07}^{+0.09}\right)$. The high-LMA solution (see, e.g. [67]) is excluded at $\sim 3.3 \sigma$. Maximal solar neutrino mixing is ruled out at $\sim 6 \sigma$; at $95 \%$ C.L. one finds $\cos 2 \theta_{\odot} \geq 0.28$ [22], which has important implications for $|\langle m\rangle|$ (see further). In the case of 3- $\nu$ mixing, the $\nu_{e}$ and $\bar{\nu}_{e}$ survival probabilities, relevant to the interpretation of the solar neutrino, KL and CHOOZ data, depend also on $\theta_{13}[21,68]$. A combined $3-\nu$ oscillation analysis of these data gives $[14,22,23]$

$$
\sin ^{2} \theta_{13}<0.027(0.047), \quad \text { at } 95 \%(99.73 \%) \text { C.L. }
$$

\footnotetext{
${ }^{8}$ For a discussion of the relevant formalism see, e.g. [19,57]. Notice that in [19] $m_{0}$ was used in the case of QD spectrum to indicate $m_{0} \equiv m_{1} \simeq m_{2} \simeq m_{3}$. Here we extend this notation to indicate the smallest neutrino mass for each type of spectrum.

${ }^{9}$ The current atmospheric neutrino data are insensitive to $\theta_{13}$ satisfying the CHOOZ limit [5].
} 
Furthermore, such an analysis shows [22] that for $\sin ^{2} \theta_{13} \lesssim 0.02$ the allowed ranges of $\Delta m_{21}^{2}$ and $\sin ^{2} \theta_{21}$ do not differ substantially from those derived in the $2-\nu$ oscillation analyses, and that the best fit values are practically independent of $\sin ^{2} \theta_{13}<0.05$. The best fit values and the allowed ranges at $95 \%$ C.L. read [14,22]:

$$
\begin{gathered}
\Delta m_{21}^{2}=8.0 \times 10^{-5} \mathrm{eV}^{2}, \quad \sin ^{2} \theta_{21}=0.31, \\
\Delta m_{21}^{2}=(7.3-8.5) \times 10^{-5} \mathrm{eV}^{2}, \quad \sin ^{2} \theta_{12}=(0.26-0.36) .
\end{gathered}
$$

Existing data allow a determination of $\Delta m_{\odot}^{2}, \sin ^{2} \theta_{\odot}$ and $\left|\Delta m_{\mathrm{A}}^{2}\right|$ at $3 \sigma$ with an error of approximately $12 \%, 24 \%$, and $50 \%$, respectively. These parameters can (and very likely will) be measured with much higher accuracy in the future. The data from phase-III of the SNO experiment [3] ${ }^{10}$ could lead to a reduction of the error in $\sin ^{2} \theta_{12}$ to $21 \%$ [29,30]. If instead of 766.3 tyr one uses simulated 3 ktyr KamLAND data in the same global solar and reactor neutrino data analysis, the $3 \sigma$ errors in $\Delta m_{21}^{2}$ and $\sin ^{2} \theta_{12}$ diminish to $7 \%$ and $18 \%$ [30]. The most precise measurement of $\Delta m_{21}^{2}$ could be achieved [29] using Super-Kamiokande doped with $0.1 \%$ of gadolinium for detection of reactor $\bar{\nu}_{e}$ [64]: the SK detector gets the same flux of reactor $\bar{\nu}_{e}$ as KamLAND and after 3 years of data-taking, $\Delta m_{21}^{2}$ could be determined with an error of $3.5 \%$ at $3 \sigma$ [29]. A dedicated reactor $\bar{\nu}_{e}$ experiment with a baseline $L \sim 60 \mathrm{~km}$, tuned to the minimum of the $\bar{\nu}_{e}$ survival probability, could provide the most precise determination of $\sin ^{2} \theta_{12}[30,65]$ : with statistics of $\sim 60 \mathrm{GW}$ ktyr and systematic error of $2 \%(5 \%), \sin ^{2} \theta_{12}$ could be measured with an error of $6 \%(9 \%)$ at $3 \sigma$ [30]. The inclusion of the uncertainty in $\theta_{13}\left(\sin ^{2} \theta_{13}<0.05\right)$ in the analyses increases the quoted errors by $(1-3) \%$ to approximately $9 \%(12 \%)$ [30]. The highest precision in the determination of $\left|\Delta m_{\mathrm{A}}^{2}\right|=\left|\Delta m_{31}^{2}\right|$ is expected to be achieved from the studies of $\nu_{\mu}$-oscillations in the T2K (SK) [69] experiment: if the true $\left|\Delta m_{31}^{2}\right|=2 \times 10^{-3} \mathrm{eV}^{2}$ (and true $\sin ^{2} \theta_{23}=0.5$ ), the $3 \sigma$ uncertainty in $\left|\Delta m_{\mathrm{A}}^{2}\right|$ is estimated to be reduced in this experiment to $\sim 12 \%$ [26]. The error diminishes with increasing $\left|\Delta m_{31}^{2}\right|$.

In what regards the CHOOZ angle $\theta_{13}$, there are several proposals for reactor $\bar{\nu}_{e}$ experiments with baseline $L \sim(1-2) \mathrm{km}$ [27], which could improve the current limit, $\sin ^{2} \theta_{13}<0.05$, by a factor of (5-10): Double-CHOOZ (in France), Braidwood (in the USA), Daya-Bay (USA-China), KASKA (in Japan), etc. The reactor $\theta_{13}$ experiments can compete in sensitivity with accelerator experiments (T2K [69], $\mathrm{NO} \nu \mathrm{A}[70]$ ) (see, e.g. [26]) and can be done on a relatively short (for experiments in this field) time scale.

Information on the absolute scale of neutrino masses can be derived in ${ }^{3} \mathrm{H} \beta$-decay experiments [71-73] and from cosmological and astrophysical data. The most stringent upper bounds on the $\bar{\nu}_{e}$ mass were obtained in the Troitzk [72] and Mainz [73] experiments:

$$
m_{\bar{\nu}_{e}}<2.3 \mathrm{eV} \text { at } 95 \% \text { C.L.. }
$$

We have $m_{\bar{\nu}_{e}} \cong m_{1,2,3}$ in the case of the QD $\nu$-mass spectrum. The KATRIN experiment [73] is planned to reach a sensitivity of $m_{\bar{\nu}_{e}} \sim 0.20 \mathrm{eV}$, i.e. it will probe the region of the QD spectrum. The Cosmic Microwave Background (CMB) data of the WMAP experiment, combined with data from large scale structure surveys (2dFGRS, SDSS), lead to an upper limit on the sum of the neutrino masses [74]:

$$
\sum_{j} m_{j} \equiv \Sigma<(0.7-2.0) \mathrm{eV} \quad \text { at } 95 \% \text { C.L. }
$$

\footnotetext{
${ }^{10}$ During this phase the neutral current rate will be measured in SNO with ${ }^{3} \mathrm{He}$ proportional counters.
} 
where we have included a conservative estimate of the uncertainty in the upper limit (see, e.g. [75]). The WMAP and future PLANCK experiments can be sensitive to $\Sigma \cong 0.4 \mathrm{eV}$. Data on weak lensing of galaxies by large scale structure, combined with data from the WMAP and PLANCK experiments, may allow $\Sigma$ to be determined with an uncertainty of $\delta \sim(0.04-0.10) \mathrm{eV}[76]$. Similar sensitivities can be reached by analysing the distortions in the Cosmic Microwave Background due to gravitational lensing in a future high sensitivity experiment [77].

\section{Predictions for the Effective Majorana Mass $|<m>|$}

Given $\Delta m_{\odot}^{2},\left|\Delta m_{\mathrm{A}}^{2}\right|, \theta_{\odot}$ and $\sin ^{2} \theta_{13}$, the value of $|<m>|$ depends strongly on the type of the neutrino mass spectrum (NH, IH, QD, etc.) and on the Majorana CPV phases of the PMNS matrix, $\alpha_{21,31}$ (see eq. (4)). In what follows we will summarise the current status of the predictions for $|<m>|$.

Normal Hierarchical Spectrum. In this case $m_{1} \ll m_{2} \ll m_{3}, m_{0}=m_{1}$, and one has $[19]$

$$
\begin{aligned}
|<m>|= & \mid\left(m_{1} \cos ^{2} \theta_{\odot}+e^{i \alpha_{21}} \sqrt{\Delta m_{\odot}^{2}+m_{1}^{2}} \sin ^{2} \theta_{\odot}\right) \cos ^{2} \theta_{13} \\
& +\sqrt{\Delta m_{\mathrm{A}}^{2}+m_{1}^{2}} \sin ^{2} \theta_{13} e^{i \alpha_{31}} \mid \\
\simeq & \left|\sqrt{\Delta m_{\odot}^{2}} \sin ^{2} \theta_{\odot} \cos ^{2} \theta_{13}+\sqrt{\Delta m_{\mathrm{A}}^{2}} \sin ^{2} \theta_{13} e^{i\left(\alpha_{31}-\alpha_{21}\right)}\right|
\end{aligned}
$$

where we have neglected $m_{1}$ in eq. (14). Although one neutrino, $\nu_{1}$, effectively "decouples" and does not contribute to $|\langle m\rangle|$, the value of $|\langle m\rangle|$ according to eq. (14) still depends on the Majorana CPV phase difference $\alpha_{32}=\alpha_{31}-\alpha_{21}$. This reflects the fact that in contrast to the case of massive Dirac neutrinos (or quarks), CP-violation can take place in the mixing of only two massive Majorana neutrinos [17].

Since at $95 \%(99.73 \%)$ C.L. we have $[14,22] \sqrt{\Delta m_{\odot}^{2}} \lesssim 9.2(9.4) \times 10^{-3} \mathrm{eV}, \sin ^{2} \theta_{\odot} \lesssim$ $0.36(0.40), \sqrt{\Delta m_{\mathrm{A}}^{2}} \lesssim 5.4(5.7) \times 10^{-2} \mathrm{eV}, \sin ^{2} \theta_{13}<0.027$ (0.046), and the largest neutrino mass enters into the expression for $|<m>|$ multiplied by the factor $\sin ^{2} \theta_{13}$, the predicted value of $|<m>|$ is typically $\sim$ few $\times 10^{-3} \mathrm{eV}$ : for $\sin ^{2} \theta_{13}=0.03$, one finds $|<m>| \lesssim 0.005 \mathrm{eV}$ (using the data at $95 \%$ C.L.). Using the best fit values of the indicated parameters (see eqs. (8) and (10) ) and the same value of $\sin ^{2} \theta_{13}=0.03$, we get $|<m>| \lesssim 0.0042 \mathrm{eV}$.

The minimal value of $|\langle m\rangle|$ in eq. (14) is obtained when there is a maximal compensation between the "solar neutrino" term, $\sqrt{\Delta m_{\odot}^{2}} \sin ^{2} \theta_{\odot} \cos ^{2} \theta_{13}$, and the "atmospheric neutrino" one, $\sqrt{\Delta m_{\mathrm{A}}^{2}} \sin ^{2} \theta_{13}$. At $95 \%$ (99.73\%) C.L. we have $\sqrt{\Delta m_{\mathrm{A}}^{2}} \sin ^{2} \theta_{13} \lesssim 1.5(2.7) \times$ $10^{-3} \mathrm{eV}$, while the "solar neutrino" term takes values in the interval $(2.1-3.2) \times 10^{-3} \mathrm{eV}$ $\left((1.9-3.6) \times 10^{-3} \mathrm{eV}\right)$. Thus, at $95 \%$ C.L. the "solar neutrino" term is larger than the "atmospheric neutrino" one and $|<m>|$ is bounded from below. However, this may not be true considering the current $99.73 \%$ C.L. intervals of allowed values of the relevant oscillation parameters. 
It follows from eq. (13) and the allowed ranges of values of $\Delta m_{\odot}^{2}, \Delta m_{\mathrm{A}}^{2}, \sin ^{2} \theta_{\odot}, \sin ^{2} \theta_{13}$, as well as of the lightest neutrino mass $m_{1}$ and CPV phases $\alpha_{21,31}$, that in the case of spectrum with normal hierarchy there can be a complete cancellation between the three terms in eq. (13), and one can have [58] $|<m>|=0$.

Inverted Hierarchical Spectrum. For IH neutrino mass spectrum, $\Delta m_{\mathrm{A}}^{2}<0, m_{0}=$ $m_{3}$, and $m_{3} \ll m_{1} \cong m_{2} \cong \sqrt{\left|\Delta m_{\mathrm{A}}^{2}\right|}=\sqrt{\Delta m_{23}^{2}}$. Using eq. (4I) we find [19,24]:

$$
\begin{aligned}
|<m>| & \cong\left|\left(\cos ^{2} \theta_{\odot}+e^{i \alpha_{21}} \sin ^{2} \theta_{\odot}\right) \cos ^{2} \theta_{13} \sqrt{m_{3}^{2}+\left|\Delta m_{\mathrm{A}}^{2}\right|}+m_{3} \sin ^{2} \theta_{13} e^{i \alpha_{31}}\right| \\
& \cong \sqrt{m_{3}^{2}+\left|\Delta m_{\mathrm{A}}^{2}\right|} \cos ^{2} \theta_{13}\left(1-\sin ^{2} 2 \theta_{\odot} \sin ^{2} \frac{\alpha_{21}}{2}\right)^{\frac{1}{2}} \\
& \cong \sqrt{\left|\Delta m_{\mathrm{A}}^{2}\right|} \cos ^{2} \theta_{13}\left(1-\sin ^{2} 2 \theta_{\odot} \sin ^{2} \frac{\alpha_{21}}{2}\right)^{\frac{1}{2}},
\end{aligned}
$$

where we have neglected $m_{3} \sin ^{2} \theta_{13}$ in eqs. (16) and (17). The term $m_{3} \sin ^{2} \theta_{13}$ can always be neglected given the existing data: even in the case where the spectrum is with partial inverted hierarchy and $m_{3}^{2} \sim\left|\Delta m_{\mathrm{A}}^{2}\right|$, the minimum of the sum of the other two terms in $|<m>|$ satisfies $\sqrt{m_{3}^{2}+\left|\Delta m_{\mathrm{A}}^{2}\right|} \cos 2 \theta_{\odot} \cos ^{2} \theta_{13} \gg m_{3} \sin ^{2} \theta_{13}$, since the data on $\theta_{\odot}$ and $\theta_{13}$ imply $\cos 2 \theta_{\odot} \gg \tan ^{2} \theta_{13}$. Even though in eqs. (16) and (17) one of the three massive Majorana neutrinos "decouples", the value of $|<m>|$ depends on the Majorana CP-violating phase $\alpha_{21}$. It follows from eq. (17) that

$$
\sqrt{\left|\Delta m_{\mathrm{A}}^{2}\right|} \cos 2 \theta_{\odot} \cos ^{2} \theta_{13} \leq|<m>| \leq \sqrt{\left|\Delta m_{\mathrm{A}}^{2}\right|} \cos ^{2} \theta_{13}
$$

The lower and upper limits correspond to the CP-conserving cases $\alpha_{21}=\pi$ and $\alpha_{21}=0$. Most remarkably, since according to the solar neutrino and KamLAND data $\cos 2 \theta_{\odot} \sim 0.40$ and $\cos 2 \theta_{\odot} \gtrsim 0.28$ at $95 \%$ C.L., we get a significant lower limit on $|<m>|$ exceeding $10^{-2} \mathrm{eV}$ in this case $[25,58]$. Using, e.g. the best fit values of $\left|\Delta m_{\mathrm{A}}^{2}\right|$ and $\sin ^{2} \theta_{\odot}$ we find: $|<m>| \gtrsim 0.02 \mathrm{eV}$. The maximal value of $|<m>|$ is determined by $\left|\Delta m_{\mathrm{A}}^{2}\right|$ and, according to eqs. (8) and (91), can reach $|<m>| \sim 0.055 \mathrm{eV}$. The indicated values of $|<m\rangle \mid$ are within the range of sensitivity of the next generation of $(\beta \beta)_{0 \nu}$-decay experiments.

Let us note that if $\Delta m_{\mathrm{A}}^{2}<0$, i.e. if the neutrino mass spectrum is with inverted hierarchy, an upper limit on $\Sigma=\left(m_{1}+m_{2}+m_{3}\right) \leq 0.10 \mathrm{eV}$ would imply $m_{3} \lesssim 0.02 \mathrm{eV}$ and therefore $m_{3}^{2} \ll\left|\Delta m_{\mathrm{A}}^{2}\right|$. In this case the spectrum would be of the IH type and eqs. (17) and (18) would be valid.

The expression for $|<m>|$, eq. (17), permits to relate the value of $\sin ^{2} \alpha_{21} / 2$ to the experimentally measurable quantities $[19,24]|<m>|, \Delta m_{\mathrm{A}}^{2}$ and $\sin ^{2} 2 \theta_{\odot}$ :

$$
\sin ^{2} \frac{\alpha_{21}}{2} \cong\left(1-\frac{|<m>|^{2}}{\left|\Delta m_{\mathrm{A}}^{2}\right| \cos ^{4} \theta_{13}}\right) \frac{1}{\sin ^{2} 2 \theta_{\odot}} .
$$

A sufficiently accurate measurement of $|<m>|$ and of $\left|\Delta m_{\mathrm{A}}^{2}\right|$ and $\theta_{\odot}$, could allow us to get information about the value of $\alpha_{21}$. If, e.g. the data show unambiguously that $|<m>|<$ $\sqrt{\left|\Delta m_{\mathrm{A}}^{2}\right|} \cos ^{2} \theta_{13}$, that would imply $\alpha_{21} \neq 0$. If in addition the data show that $|<m>|>$ $\sqrt{\left|\Delta m_{\mathrm{A}}^{2}\right|} \cos 2 \theta_{\odot} \cos ^{2} \theta_{13}$, one could conclude that $\alpha_{21}$ takes a CP-violating value. 
Three Quasi-Degenerate Neutrinos. In this case $m_{0} \equiv m_{1} \cong m_{2} \cong m_{3}, m_{0}^{2} \gg$ $\left|\Delta m_{\mathrm{A}}^{2}\right|, m_{0} \gtrsim 0.10 \mathrm{eV}$. Hence, $|<m>|$ is essentially independent of $\Delta m_{\mathrm{A}}^{2}$ and $\Delta m_{\odot}^{2}$, and the two possibilities, $\Delta m_{\mathrm{A}}^{2}>0$ and $\Delta m_{\mathrm{A}}^{2}<0$, lead effectively to the same predictions for $|<m>|$. The mass $m_{0}$ coincides with the $\bar{\nu}_{e}$ mass $m_{\bar{\nu}_{e}}$ measured in the ${ }^{3} \mathrm{H} \beta$-decay experiments: $m_{0}=m_{\bar{\nu}_{e}}$. Thus, $m_{0}<2.3 \mathrm{eV}$, or if we use a conservative cosmological upper limit [75], $m_{0}=\Sigma / 3<0.7 \mathrm{eV}$. The QD $\nu$-mass spectrum is realized for values of $m_{0}$, that can be measured in the ${ }^{3} \mathrm{H} \beta$-decay experiment KATRIN [73]. The effective Majorana mass $|<m>|$ is given by

$$
\begin{aligned}
|<m>| & \cong m_{0}\left|\left(\cos ^{2} \theta_{\odot}+\sin ^{2} \theta_{\odot} e^{i \alpha_{21}}\right) \cos ^{2} \theta_{13}+e^{i \alpha_{31}} \sin ^{2} \theta_{13}\right| \\
& \cong m_{0}\left|\cos ^{2} \theta_{\odot}+\sin ^{2} \theta_{\odot} e^{i \alpha_{21}}\right|=m_{0} \sqrt{1-\sin ^{2} 2 \theta_{\odot} \sin ^{2} \frac{\alpha_{21}}{2}}
\end{aligned}
$$

Similarly to the case of the IH spectrum, one has:

$$
m_{0} \cos 2 \theta_{\odot} \lesssim|<m>| \leq m_{0} .
$$

For $\cos 2 \theta_{\odot} \sim 0.40$, favored by the data, one finds a non-trivial lower limit on $|<m>|$, $|<m>| \gtrsim 0.08 \mathrm{eV}$. For values of the parameters allowed at $95 \%$ C.L. one has $|<m\rangle \mid \gtrsim$ $0.06 \mathrm{eV}$. Using the conservative cosmological upper bound on $\Sigma$ we get $|<m>|<0.70 \mathrm{eV}$. Also in this case one can obtain, in principle, direct information on one CPV phase from the measurement of $|<m>|, m_{0}$ and $\sin ^{2} 2 \theta_{\odot}$ :

$$
\sin ^{2} \frac{\alpha_{21}}{2} \cong\left(1-\frac{|<m>|^{2}}{m_{0}^{2}}\right) \frac{1}{\sin ^{2} 2 \theta_{\odot}} .
$$

The specific features of the predictions for $|\langle m\rangle|$ in the cases of the three types of neutrino mass spectrum discussed above are evident in Fig. 1, where the dependence of $|<m>|$ on $m_{0}=\min \left(m_{j}\right)$ for $\sin ^{2} \theta_{\odot}=0.31$ and $\sin ^{2} \theta_{13}=0.01$ and 0.03 is shown. The figures are obtained by including a $2 \sigma$ uncertainty in the predicted value of $|<m>|$. The uncertainty in $|<m>|, \sigma(|<m>|)$, has been calculated by exploiting the explicit dependence of $|<m>|$ on the oscillation parameters $\Delta m_{\odot}^{2}, \Delta m_{\mathrm{A}}^{2}, \sin ^{2} \theta_{\odot}$ and $\sin ^{2} \theta_{13}$ and assuming the following $1 \sigma$ errors (achievable in the future) in the determination of the latter: $\sigma\left(\Delta m_{\odot}^{2}\right)=2 \%$, $\sigma\left(\left|\Delta m_{\mathrm{A}}^{2}\right|\right)=6 \%, \sigma\left(\sin ^{2} \theta_{12}\right)=4 \%$ and two values of $\sigma\left(\sin ^{2} \theta_{13}\right)=0.004$ and 0.008 . The current best fit values of $\Delta m_{\odot}^{2}$ and $\left|\Delta m_{\mathrm{A}}^{2}\right|$ have been used. The Majorana CP-violation phases $\alpha_{21}$ and $\alpha_{31}$ were varied over all possible values they can take ${ }^{11}$. For the NH and QD (and interpolating) spectra, the regions within the black lines of a given type (solid, short-dashed, long-dashed, dash-dotted) correspond to the four different sets of CP-conserving values of the two phases $\alpha_{21}$ and $\alpha_{31}$, and thus to the four possible combinations of the relative CP parities $\left(\eta_{21}, \eta_{31}\right)$ of neutrinos $\nu_{1,2}$ and $\nu_{1,3}:(+1,+1)$ solid, $(-1,-1)$ short-dashed, $(+1,-1)$ long-dashed, and $(-1,+1)$ dash-dotted lines. If the spectrum is IH, the contribution to $|<m>|$ due to $m_{3}$ can be neglected and the predictions for $|<m>|$ become practically independent of $\alpha_{31}\left(\eta_{31}\right)$. In this case the regions delimited by the black solid (dotted) lines correspond to $\eta_{21}=+1\left(\eta_{21}=-1\right)$. In the case of $\mathrm{CP}$-violation all colored regions are allowed.

\footnotetext{
${ }^{11}$ It follows from eq. (41) that $|\langle m\rangle|$ is symmetric under the transformations $\alpha_{21,31} \rightarrow 2 \pi-\alpha_{21,31}$. This implies that it is sufficient to consider values of $\alpha_{21,31}$ in the range $[0, \pi]$ to cover all possible physical configurations for $|<m>|$.
} 

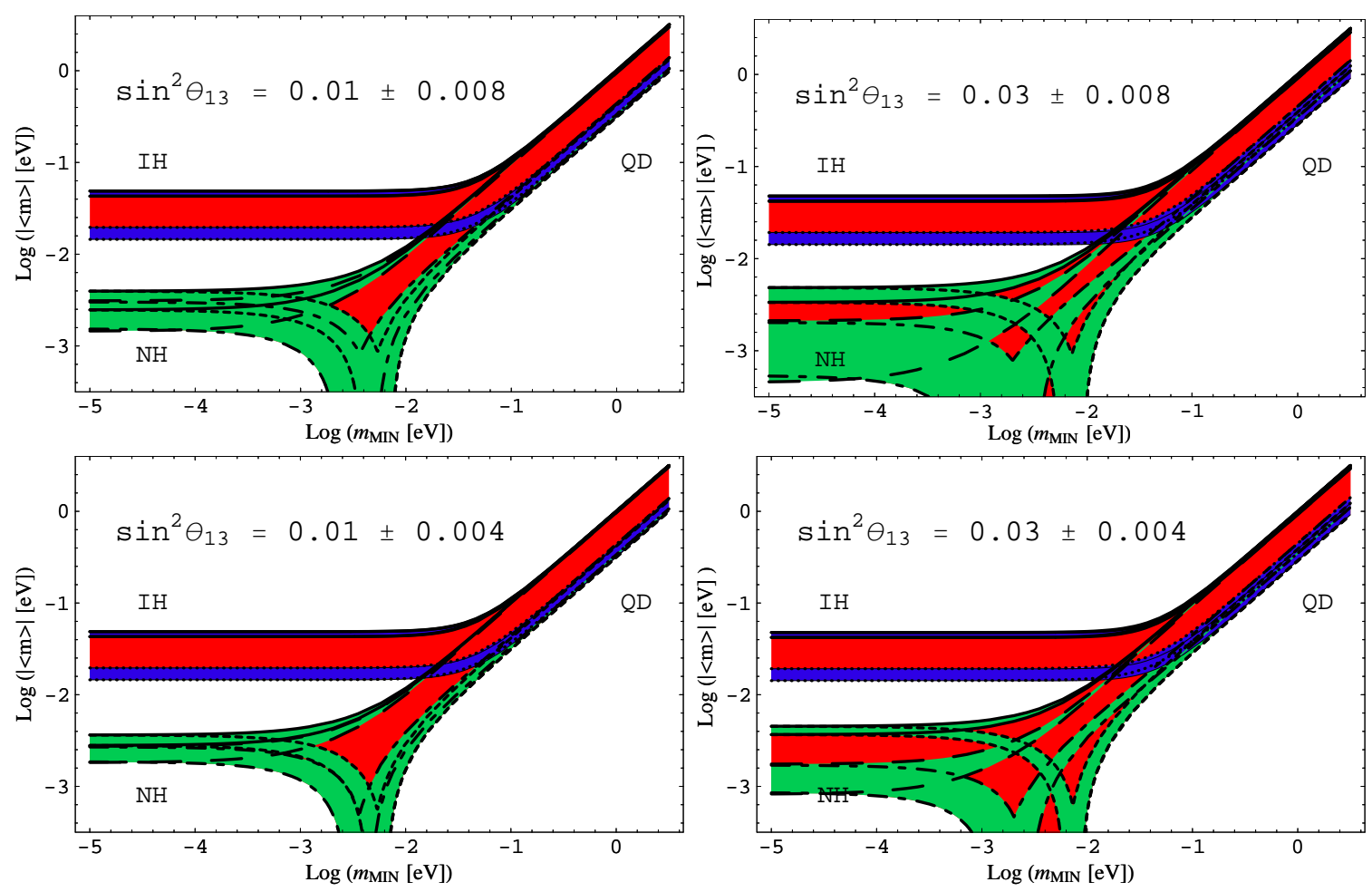

Figure 1: The predicted value of $|<m>|$ (including a prospective $2 \sigma$ uncertainty) as a function of $\min \left(m_{j}\right)$ for $\sin ^{2} \theta_{\odot}=0.31$ and $\sin ^{2} \theta_{13}=0.01 ; 0.03$, and two different assumptions on the error on $\sin ^{2} \theta_{13}$. For the NH and QD (and interpolating) spectra, the regions within the black lines of a given type (solid, short-dashed, long-dashed, dash-dotted) correspond to the four different sets of CP-conserving values of the two phases $\alpha_{21}$ and $\alpha_{31}$, and thus to the four possible combinations of the relative CP parities $\left(\eta_{21}, \eta_{31}\right)$ of neutrinos $\nu_{1,2}$ and $\nu_{1,3}:(+1,+1)$ solid, $(-1,-1)$ short-dashed, $(+1,-1)$ long-dashed, and $(-1,+1)$ dash-dotted lines. For the IH spectrum, the regions delimited by the black solid (dotted) lines correspond to $\eta_{21}=+1$ $\left(\eta_{21}=-1\right)$, independently of $\eta_{31}$. The regions shown in red/medium-gray correspond to violation of CP-symmetry (see text for further details).

The regions shown in red/medium-gray are the so-called "just CP-violation" regions [19]: an experimental point in these regions would unambiguously signal CP-violation associated with Majorana neutrinos. In the regions shown in blue/dark-gray and in green/light-gray it is not possible to distinguish between CP-violation and CP-conservation, because of the uncertainty implied by the errors on the oscillation parameters.

The impact the prospective errors on $\Delta m_{\mathrm{A}}^{2}, \Delta m_{\odot}^{2}$ and $\sin ^{2} \theta_{\odot}$ have on the predictions for $|<m>|$ is, in general, very small. More specifically, in the case of QD spectrum, the contributions of $\sigma\left(\Delta m_{\mathrm{A}}^{2}\right), \sigma\left(\Delta m_{\odot}^{2}\right)$ and $\sigma\left(\sin ^{2} \theta_{13}\right)$ in $\sigma(|<m>|)$ can be neglected and only $\sigma\left(\sin ^{2} \theta_{\odot}\right)$ induces an uncertainty in $|<m>|$ which can be as large as few $\%$ if $\alpha_{21} \sim \pi$. For the IH type of spectrum, both $\sigma\left(\left|\Delta m_{\mathrm{A}}^{2}\right|\right)$ and $\sigma\left(\sin ^{2} \theta_{12}\right)$ are relevant and contribute to an overall $\sigma(|<m>|) \sim$ several \%. Also in this case the error on $|<m>|$ due to $\sigma\left(\sin ^{2} \theta_{12}\right)$ increases as $\alpha_{21}$ varies from 0 to $\pi$. For $\mathrm{NH}$ spectrum, the dominant source of error is $\sigma\left(\sin ^{2} \theta_{13}\right)$. It affects significantly the predicted value of $|\langle m\rangle|$. This explains the different allowed ranges of values for $|<m>|$ obtained for $\sigma\left(\sin ^{2} \theta_{13}\right)=0.004$ and 0.008 . Notice that 
the impact of the errors is larger the smaller $|<m>|$ is, i.e. when $\left(\alpha_{31}-\alpha_{21}\right)$ approaches the value $\pi$.

If the spectrum is with normal hierarchy $\left(\Delta m_{\mathrm{A}}^{2}>0\right),|<m>|$ can lie anywhere between 0 and the currently existing upper limits, eqs. (6) and (7). This conclusion does not change even under the most favorable conditions for the determination of $|<m\rangle \mid$, namely, even when $\left|\Delta m_{\mathrm{A}}^{2}\right|, \Delta m_{\odot}^{2}, \theta_{\odot}$ and $\theta_{13}$ are known with negligible uncertainty.

The "gap" between the predicted values of $|\langle m\rangle|$ in the cases of IH and NH spectra allows us, in principle, to distinguish between these two types of hierarchical spectra [25,58]. Establishing, for instance, that $|<m>| \neq 0$ but $|<m>|<10^{-2} \mathrm{eV}$ would imply, within the 3-neutrino mixing scheme with Majorana neutrinos under discussion, that the neutrino mass spectrum is with normal hierarchy, i.e. $\Delta m_{\mathrm{A}}^{2}>0$. Depending on the value of $m_{1}$, the spectrum could be either normal hierarchical (NH) or with partial hierarchy [19]. Obviously, such a result would rule out both the IH and QD spectrum.

If the results in [53] implying $|\langle m\rangle|=(0.1-0.9) \mathrm{eV}$ are confirmed, this would mean, in particular, that the neutrino mass spectrum is of the QD type. In this case, however, the measurement of $|\langle m\rangle|$ cannot provide information on the $\operatorname{sgn}\left(\Delta m_{\mathrm{A}}^{2}\right)$.

It should be clear from the preceding discussion that, depending on the measured value of $|<m>| \neq 0$, the $(\beta \beta)_{0 \nu}$-decay experiments may or may not provide information on both the type of $\nu$ mass spectrum (NH, IH, QD, etc.) and $\operatorname{sgn}\left(\Delta m_{\mathrm{A}}^{2}\right)$. If $|<m>| \sim$ few $\times$ $10^{-3} \mathrm{eV}<10^{-2} \mathrm{eV}$, both the type of the spectrum and $\operatorname{sgn}\left(\Delta m_{\mathrm{A}}^{2}\right)$ will be determined. For $\sqrt{\left|\Delta m_{\mathrm{A}}^{2}\right|} \cos 2 \theta_{\odot} \leq|<m>| \leq \sqrt{\left|\Delta m_{\mathrm{A}}^{2}\right|}$, it would be possible to conclude that $\operatorname{sgn}\left(\Delta m_{\mathrm{A}}^{2}\right)<$ 0 only if $m_{0} \lesssim 0.02 \mathrm{eV}$, i.e. $m_{0}^{2} \ll\left|\Delta m_{\mathrm{A}}^{2}\right|$. In a relatively narrow interval of values of $m_{0} \sim$ few $\times 10^{-2} \mathrm{eV}$, for which $m_{0}^{2} \sim\left|\Delta m_{\mathrm{A}}^{2}\right|$, one can have both $\Delta m_{\mathrm{A}}^{2}<0$ and $\Delta m_{\mathrm{A}}^{2}>0$. In the latter case the $\nu$ mass spectrum is with partial hierarchy. If $|\langle m\rangle| \gtrsim 0.10 \mathrm{eV}$, the $\nu$ mass spectrum is $\mathrm{QD}$ and the measurement of $|\langle m\rangle|$ will provide no information on $\operatorname{sgn}\left(\Delta m_{\mathrm{A}}^{2}\right)$.

Finally, if neutrino oscillation experiments show that $\Delta m_{\mathrm{A}}^{2}<0$ and therefore the $\nu$ mass spectrum is with inverted hierarchy, while in $(\beta \beta)_{0 \nu}$-decay experiments only the upper limit $|<m>|<\sqrt{\left|\Delta m_{\mathrm{A}}^{2}\right|} \cos 2 \theta_{\odot} \cos ^{2} \theta_{13}$ is obtained, that would mean either that there is a new additional contribution to the $(\beta \beta)_{0 \nu}$-decay amplitude which interferes destructively with that due to the light Majorana neutrino exchange, or that the massive neutrinos $\nu_{j}$ are Dirac particles. Similar conclusion could be made if, e.g., the KATRIN experiment shows that $m_{0} \gtrsim 0.2 \mathrm{eV}$ and correspondingly the $\nu$ mass spectrum is $\mathrm{QD}$, while $(\beta \beta)_{0 \nu}$-decay experiments demonstrate only that the upper limit $|<m>|<m_{0} \cos 2 \theta_{\odot}$ holds.

\section{Analysis of the Implications of a $(\beta \beta)_{0 \nu}$-Decay Half- Life Measurement}

\subsection{On the NME Uncertainties}

If the $(\beta \beta)_{0 \nu^{-}}$-decay of a given nucleus is observed, it will be possible to determine the value of $|\langle m\rangle|$ from the measurement of the associated half-life of the decay. This would require the knowledge of the nuclear matrix element of the process. At present there exist large uncertainties in the calculation of the $(\beta \beta)_{0 \nu}$-decay nuclear matrix elements (see, 
e.g. [49]). This is reflected, in particular, in the factor of $\sim 3$ uncertainty in the upper limit on $|<m>|$, which is extracted from the experimental lower limits on the $(\beta \beta)_{0 \nu}$-decay half-life of ${ }^{76} \mathrm{Ge} .{ }^{12}$ For some nuclei (such as ${ }^{100} \mathrm{Mo},{ }^{130} \mathrm{Te},{ }^{136} \mathrm{Xe}$ ), the uncertainties can be even larger. Recently, encouraging results on the problem of calculating the nuclear matrix elements have been obtained in [78]. A discussion of the problems related to the calculation of the $(\beta \beta)_{0 \nu}$-decay NME is outside the scope of the present work. We would like to only note here that the observation of a $(\beta \beta)_{0 \nu}$-decay of one nucleus is likely to lead to searches and eventually to observation of the decay of other nuclei. It can be expected that such a progress will help, in particular, to solve the problem of the sufficiently precise calculation of the nuclear matrix elements for the $(\beta \beta)_{0 \nu}$-decay [79].

\subsection{The Method of Analysis}

The experimental observable in $(\beta \beta)_{0 \nu}$-decay is the decay rate $\Gamma_{\text {obs }}$ measured with an experimental accuracy $\sigma\left(\Gamma_{\text {obs }}\right)$. The observed decay rate has to be compared with the theoretically predicted rate

$$
\Gamma_{\text {th }}=G|\mathcal{M}|^{2}(|<m>|(\boldsymbol{x}))^{2},
$$

where $G$ is a known phase space factor and $\mathcal{M}$ is the NME. In eq. (24) $\boldsymbol{x}=\left(\boldsymbol{x}_{\mathrm{osc}}, \boldsymbol{x}_{\beta \beta}^{0 \nu}\right)$ are the parameters determining $|<m>|$, which we divide into parameters measured in oscillation experiments, whose values we are going to use as input in the analysis,

$$
\boldsymbol{x}_{\mathrm{osc}}=\left(\theta_{12}, \theta_{13},\left|\Delta m_{31}^{2}\right|, \Delta m_{21}^{2}\right),
$$

and parameters that are, in principle, accessible by $(\beta \beta)_{0 \nu}$-decay experiments,

$$
\boldsymbol{x}_{\beta \beta}^{0 \nu}=\left(m_{0}, \operatorname{sgn}\left(\Delta m_{31}^{2}\right), \alpha_{21}, \alpha_{31}\right) .
$$

To investigate the potential to get information on the parameters $\boldsymbol{x}_{\beta \beta}^{0 \nu}$ from the result of a generic $(\beta \beta)_{0 \nu}$-decay experiment, we convert the observed decay rate and the experimental error into an "observed effective Majorana mass" and its error by

$$
|<m>|^{\mathrm{obs}} \equiv \sqrt{\frac{\Gamma_{\mathrm{obs}}}{G}} \frac{1}{\left|\mathcal{M}_{0}\right|}, \quad \sigma_{\beta \beta}=\frac{1}{2} \frac{1}{\sqrt{\Gamma_{\mathrm{obs}} G}} \frac{1}{\left|\mathcal{M}_{0}\right|} \sigma\left(\Gamma_{\mathrm{obs}}\right),
$$

where $\left|\mathcal{M}_{0}\right|$ is some nominal (theoretically predicted) value of the NME. If $|<m>|^{\text {obs }}>n \sigma_{\beta \beta}$, a positive $(\beta \beta)_{0 \nu}$-decay signal is observed at the $n \sigma$ C.L. Otherwise only an upper bound on $|<m>|$ is obtained. The quantity $\sigma_{\beta \beta}$ defined in eq. (27) is a measure for the "accuracy" of the experiment. Then we construct a $\chi^{2}$ in the following way:

$$
\chi^{2}\left(\boldsymbol{x}_{\beta \beta}^{0 \nu}, F\right)=\min _{\xi \in[1 / \sqrt{F}, \sqrt{F}]} \frac{\left[\xi|<m>|(\boldsymbol{x})-|<m>|^{\mathrm{obs}}\right]^{2}}{\sigma_{\beta \beta}^{2}+\xi^{2} \sigma_{\mathrm{th}}^{2}} .
$$

\footnotetext{
${ }^{12}$ For the uncertainty on the NME for the $(\beta \beta)_{0 \nu}$-decay half-life of ${ }^{76} \mathrm{Ge}$ commonly a factor of 10 is adopted. Since $|<m>|$ depends on the square-root of the half-life, typical values for the current uncertainty on $|<m>|$ are factors from 3 to 4 .
} 
The parameter $\xi$ takes into account the uncertainty on the NME, and it is defined by

$$
\xi \equiv \frac{|\mathcal{M}|}{\left|\mathcal{M}_{0}\right|}
$$

where $|\mathcal{M}|$ is the unknown true value of the NME and $\left|\mathcal{M}_{0}\right|$ is the nominal value used in eq. (27) to obtain $|<m>|^{\text {obs }}$. The theoretical error $\sigma_{\text {th }}$ in eq. (28) takes into account the uncertainty implied by the errors on the oscillation parameters; it is calculated by

$$
\sigma_{\mathrm{th}}^{2}=\sigma_{\mathrm{th}}^{2}\left(\boldsymbol{x}_{\mathrm{osc}}\right)=\sum_{i}\left(\frac{\partial|<m>|}{\partial x_{\mathrm{osc}}^{i}}\right)^{2}\left(\delta x_{\mathrm{osc}}^{i}\right)^{2},
$$

where the index $i$ runs over the four oscillation parameters given in eq. (25), $\delta x_{\text {osc }}^{i}$ is the uncertainty on the parameter $x_{\mathrm{osc}}^{i}$, and we have used the fact that to very good approximation the errors on the oscillation parameters are uncorrelated (see, e.g. [14]).

Assuming that the value of the NME is known within a factor $F \geq 1$, for given parameters $\boldsymbol{x}_{\beta \beta}$ we minimise the right-hand side of eq. (28) with respect to $\xi$, allowing $\xi$ to vary within the interval $[1 / \sqrt{F}, \sqrt{F}]$. A perfectly known NME corresponds to $F=1$. Note that in this way we do not introduce a probability weight for the NME; all values between $\left|\mathcal{M}_{0}\right| / \sqrt{F}$ and $\sqrt{F}\left|\mathcal{M}_{0}\right|$ are treated on an equal footing. This procedure is similar to the "flat priors" used in unitarity triangle fits of the CKM matrix in order to account for theoretical uncertainties, see e.g. [80]. We have adopted this method, since it is not possible to assign a well defined probability distribution to the parameter $\xi$, and therefore, specifying a range for $\xi$ without imposing any further weight seems to be the most reliable procedure. Since the choice of the NME uncertainty factor $F$ is subject to some arbitrariness we shall show results for various values of $F$.

To combine a measurement of the $(\beta \beta)_{0 \nu}$-decay rate with a constraint on the sum of the neutrino masses $\Sigma$ (obtained, e.g. from cosmological/astrophysical observations), we generalise eq. (28) in a straightforward way. To take into account the correlations between $|<m>|$ and $\Sigma$ induced by the uncertainties on $\Delta m_{21}^{2}$ and $\Delta m_{31}^{2}$, we use the following covariance matrix in the $\chi^{2}$-analysis:

$$
S_{a b}=\delta_{a b}\left(\sigma_{a}^{\exp }\right)^{2}+\sum_{i} \frac{\partial T_{a}}{\partial x_{\mathrm{osc}}^{i}} \frac{\partial T_{b}}{\partial x_{\mathrm{osc}}^{i}}\left(\delta x_{\mathrm{osc}}^{i}\right)^{2}, \quad a, b=1,2,
$$

where $T_{1} \equiv \xi|<m>|, T_{2} \equiv \Sigma$, and $\sigma_{1}^{\exp } \equiv \sigma_{\beta \beta}$ and $\sigma_{2}^{\text {exp }} \equiv \sigma_{\Sigma}$ are the experimental errors on $|<m>|^{\text {obs }}$ and $\Sigma$, respectively.

\subsection{Constraining the Lightest Neutrino Mass}

We start the quantitative evaluation of the physics potential of a $(\beta \beta)_{0 \nu}$-decay observation by discussing the information that can be obtained on the absolute value of the lightest neutrino mass $m_{0}$. Given an experimental result on $|<m\rangle \mid$ from a $(\beta \beta)_{0 \nu}$-decay experiment, one can infer an allowed range for $m_{0}$ for each type of neutrino mass ordering. The results of such an analysis are shown in Fig. 2. For given values of $|\langle m\rangle|^{\text {obs }}$ and its experimental error $\sigma_{\beta \beta}$, we minimize the $\chi^{2}$ of eq. (28) with respect to the phases $\alpha_{21}$ and $\alpha_{31}$, and calculate 
the allowed range for $m_{0}$ at $2 \sigma$ by using the condition $\chi^{2}\left(m_{0}\right) \leq 4$. In Fig. 2 we adopted the best fit values for $\Delta m_{21}^{2},\left|\Delta m_{31}^{2}\right|$ and $\sin ^{2} \theta_{12}$ (see eqs. (8) and (10)), and $\sin ^{2} \theta_{13}=0$. We have verified that the results hardly change if the values of the oscillation parameters are varied within the present $3 \sigma$ ranges. The dashed lines in Fig. 2 correspond to the current uncertainties of $\Delta m_{21}^{2},\left|\Delta m_{31}^{2}\right|, \sin ^{2} \theta_{12}$ and $\sin ^{2} \theta_{13}$, while the solid lines are obtained using the following prospective $1 \sigma$ errors: $\sigma\left(\Delta m_{21}^{2}\right)=2 \%, \sigma\left(\left|\Delta m_{31}^{2}\right|\right)=5 \%, \sigma\left(\sin ^{2} \theta_{12}\right)=3 \%$ and $\sigma\left(\sin ^{2} \theta_{13}\right)=0.002$. By comparing the dashed and solid lines in Fig. 2 one observes that improving the accuracy of the oscillation parameters has only a minor impact on the results: we find only small improvements of the constraints on $m_{0}$, while the qualitative behavior is unchanged. Consider first the case of $|\langle m\rangle|^{\text {obs }}=0.2 \mathrm{eV}$, shown in the right column of Fig. 2, In this case a positive signal should be established with high confidence by the next generation of $(\beta \beta)_{0 \nu}$-experiments. If the experimental error in $|<m>|^{\text {obs }}$ is sufficiently small $\left(\sigma_{\beta \beta} \lesssim 0.06 \mathrm{eV}\right.$ for NME uncertainty factor $\left.F \leq 3\right)$, i) the NH and IH spectra will be excluded and hence, the neutrino mass spectrum will be proved to be QD, ii) $m_{0}$ will be constrained to lie in a rather narrow interval of values limited from below by $m_{0} \gtrsim 0.1 \mathrm{eV}$, and iii) no information on $\operatorname{sgn}\left(\Delta m_{31}^{2}\right)$ will be obtained. The uncertainty in the NME directly translates into an uncertainty in $m_{0}$.

In the case of an "intermediate" value of $|\langle m\rangle|^{\text {obs }}=0.04 \mathrm{eV}$ shown in the middle column of Fig. 2] a lower and an upper bound on $m_{0}$ can be established for $\Delta m_{31}^{2}>0$ if $\sigma_{\beta \beta} \lesssim 0.017 \mathrm{eV}: 0.01 \mathrm{eV} \lesssim m_{0} \lesssim 0.1 \mathrm{eV}$. In the case of $\Delta m_{31}^{2}<0$ only an upper bound will be obtained: $m_{0} \lesssim 0.1 \mathrm{eV}$. This result can be easily understood from Fig. 1] if $|<m>|$ is sufficiently large and $\sigma_{\beta \beta}$ is small enough, the branch corresponding to the normal hierarchical spectrum extending to $m_{0}=0$ can be excluded.

Consider finally the left column of plots in Fig. 2 corresponding to a very small value of $|<m>|^{\text {obs }}=4 \times 10^{-3} \mathrm{eV}$. For estimated typical values of $\sigma_{\beta \beta}$ of the next generation of $(\beta \beta)_{0 \nu}$-decay experiments and the mean value of $|\langle m\rangle|$ considered, only an upper bound on $|<m>|$ can be established. It is clear that in this case one gets also only an upper bound on $m_{0}$. Moreover, from the panel corresponding to a known NME $(F=1)$ one observes that for (ambitious) experimental accuracies, i.e. for $\sigma_{\beta \beta} \lesssim 7 \times 10^{-3} \mathrm{eV}$, the case of $\operatorname{sgn}\left(\Delta m_{31}^{2}\right)<$ 0 (inverted mass hierarchy) can, in principle, be excluded. This is a consequence of the lower bound on $|\langle m\rangle|$ for inverted ordering, which follows from the fact that $\cos 2 \theta_{12}$ is significantly different from zero (see eq. (18) and the related discussion). However, if we take into account a possible uncertainty in the NME, the requirements on the experimental accuracy of $|<m>|$ become exceedingly demanding $\left(\sigma_{\beta \beta} \lesssim 4 \times 10^{-3} \mathrm{eV}\right.$ for $\left.F=2\right)$, which renders the exclusion of the neutrino mass spectrum with inverted hierarchy remarkably challenging. Reducing the error to $\sigma_{\beta \beta} \cong 10^{-3} \mathrm{eV}$ would allow, e.g. for $F \leq 2$, to conclude that $m_{0} \lesssim 0.02 \mathrm{eV}$ and the neutrino mass spectrum is normal hierarchical. Establishing in an independent experiment that $\operatorname{sgn}\left(\Delta m_{31}^{2}\right)<0$ would imply in the case under consideration that there are additional mechanism(s) of $(\beta \beta)_{0 \nu^{-}}$decay [81] whose contribution to the $(\beta \beta)_{0 \nu^{-}}$ decay amplitude compensates partially the one due to the Majorana neutrino exchange.

\subsection{Determining the Type of Neutrino Mass Spectrum}

As is clear from the previous discussions, $(\beta \beta)_{0 \nu}$-decay experiments provide a unique possibility to obtain information on the type of neutrino mass spectrum, i.e. to distinguish 


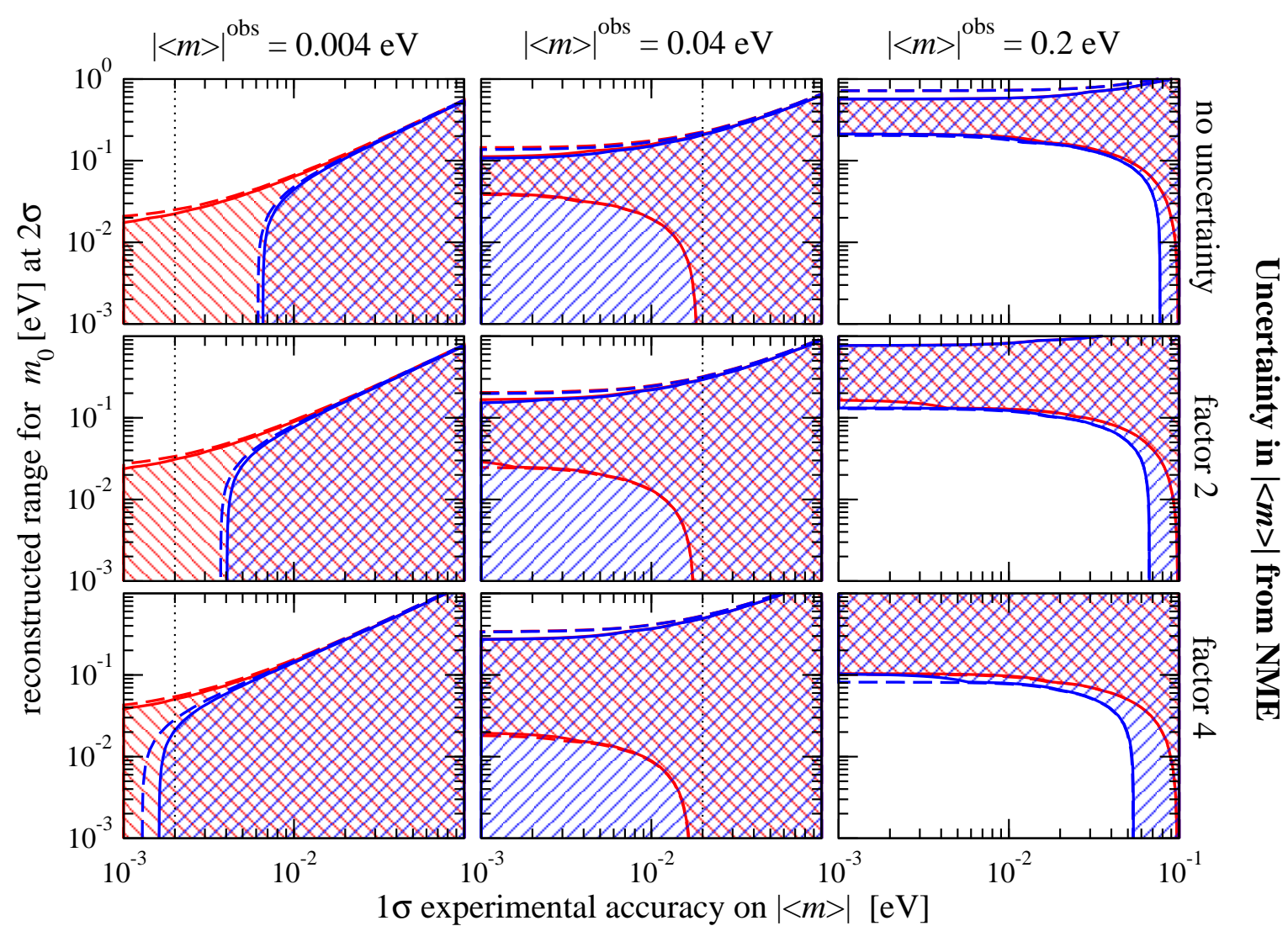

(N) normal ordering $\square Z$ inverted ordering

dashed: $\sigma\left(\sin ^{2} \theta_{13}\right)=0.016, \sigma\left(\sin ^{2} \theta_{12}\right)=7.5 \%, \sigma\left(\Delta m_{21}^{2}\right)=4 \%, \sigma\left(\Delta m_{31}^{2}\right)=13 \%$

solid: $\quad \sigma\left(\sin ^{2} \theta_{13}\right)=0.002, \sigma\left(\sin ^{2} \theta_{12}\right)=3.0 \%, \sigma\left(\Delta m_{21}^{2}\right)=2 \%, \sigma\left(\Delta m_{31}^{2}\right)=5 \%$

$$
\begin{aligned}
& \sin ^{2} \theta_{13}=0 \\
& \sin ^{2} \theta_{12}=0.31
\end{aligned}
$$

Figure 2: The reconstructed range for the lightest neutrino mass $m_{0}$ at $2 \sigma$ C.L. for normal $\left(\Delta m_{31}^{2}>0\right)$ and inverted $\left(\Delta m_{31}^{2}<0\right)$ mass ordering as a function of the $1 \sigma$ experimental error on $|<m>|^{\text {obs }}$. The results are shown for three representative values $|<m>|^{\text {obs }}=$ $0.004,0.04,0.2 \mathrm{eV}$ (columns of panels), and for fixed NME (first row), and an uncertainty of a factor of $F=2$ and $F=4$ in the NME (second and third rows). The figure is obtained using the current best fit values of $\Delta m_{21}^{2},\left|\Delta m_{31}^{2}\right|$ and $\sin ^{2} \theta_{12}$ (eqs. (8) and (10)), and $\sin ^{2} \theta_{13}=0$. The dashed (solid) lines correspond to the present (prospective) uncertainties on the oscillation parameters. To the left of the dotted lines, a positive signal is obtained at $2 \sigma$, whereas to the right only an upper bound can be stated.

between the NH, IH and QD spectra. As we have commented earlier, getting information on the possible hierarchical structure of the neutrino mass spectrum and on $\operatorname{sgn}\left(\Delta m_{31}^{2}\right)$ are different, although not totally unrelated, problems. In this subsection we elaborate further on the issue, since a determination of the neutrino mass spectrum is fundamental for our understanding of neutrino mixing. We investigate what conclusions can be drawn at the $2 \sigma$ C.L. on the neutrino mass spectrum from a result of a $(\beta \beta)_{0 \nu}$-decay experiment, characterised by the observed value of $|\langle m\rangle|$ and its experimental error. For given values of $|<m>|^{\text {obs }}, \sigma_{\beta \beta}$, the uncertainty $F$ in the NME and a fixed neutrino mass ordering, we 

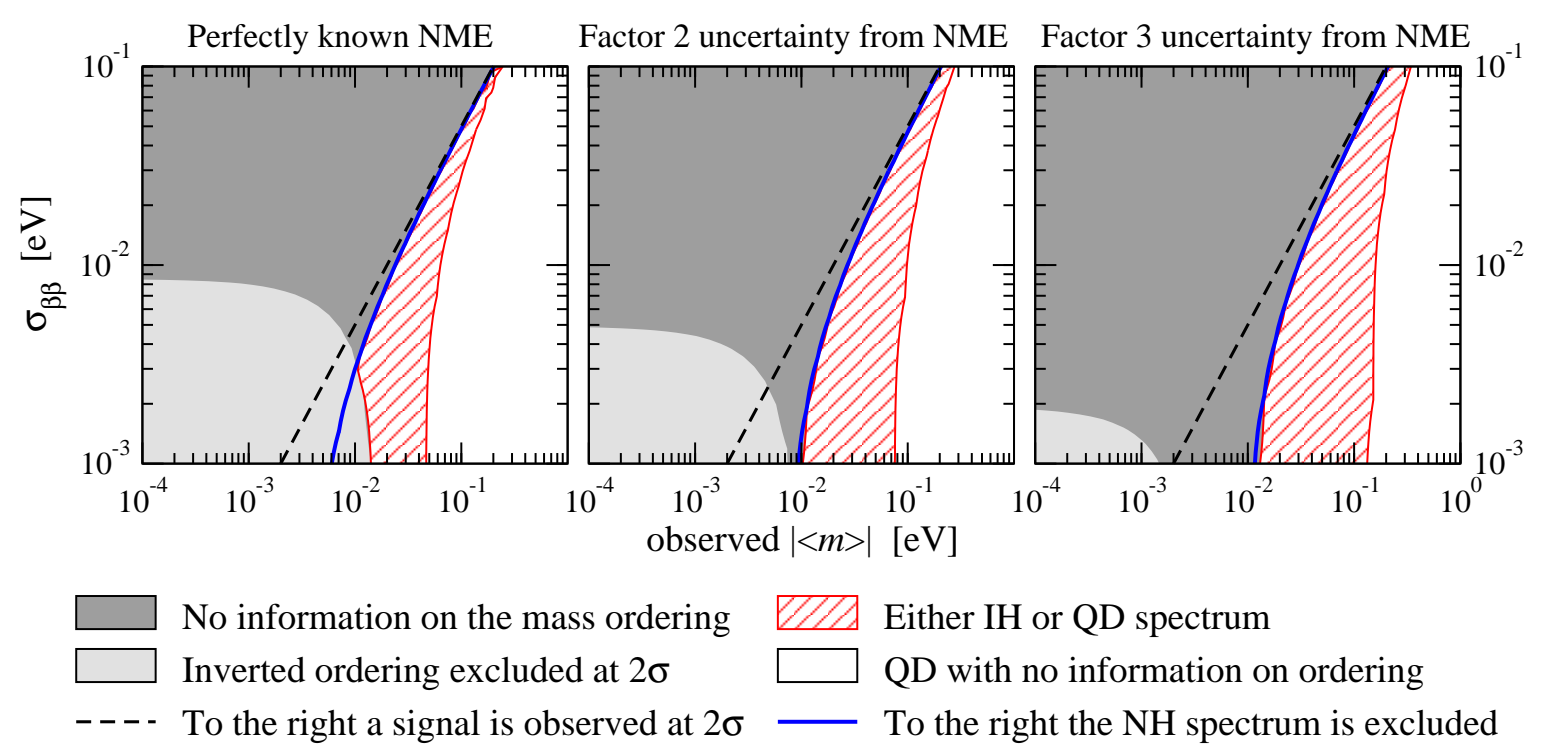

$$
\sin ^{2} \theta_{13}=0.03 \pm 0.006, \sin ^{2} \theta_{12}=0.31 \pm 3 \%, \Delta m_{21}^{2}=8 \times 10^{-5} \pm 2 \%,\left|\Delta m_{31}^{2}\right|=2.2 \times 10^{-3} \pm 3 \%
$$

Figure 3: Information on the type of neutrino mass spectrum, inferred from data on $(\beta \beta)_{0 \nu^{-}}$ decay as a function of the observed $|\langle m\rangle|$ and its experimental error for three different assumptions on the NME uncertainty factor $F$ (see text for details).

minimize the $\chi^{2}$-function of eq. (28) with respect to $m_{0}, \alpha_{21}$ and $\alpha_{31}$. If the $\chi^{2}$-minimum is smaller than 4 , we conclude that this type of ordering is allowed. In addition we test if the "data" are consistent with negligible $m_{0}$, which implies a hierarchical spectrum (more precisely, we test whether $\left.\chi^{2}\left(m_{0}=0\right) \leq 4\right)$. The results of our analysis are shown graphically in Fig. 3. For values of $|<m>|^{\text {obs }}$ and $\sigma_{\beta \beta}$ forming the dark shaded and white areas in the three panels, no information on $\operatorname{sgn}\left(\Delta m_{31}^{2}\right)$ can be obtained. The light shaded regions correspond to the case where $\operatorname{sgn}\left(\Delta m_{31}^{2}\right)<0$ (inverted mass ordering) can be excluded. In agreement with the results presented in the previous subsection, we find that this is only possible for $|<m>|^{\text {obs }}<0.01 \mathrm{eV}$ and an experimental error well below $0.01 \mathrm{eV}$. To the right of the solid curve, the spectrum cannot be hierarchical for $\operatorname{sgn}\left(\Delta m_{31}^{2}\right)>0$, i.e. the possibility $m_{1} \ll m_{2} \ll m_{3}$ is ruled out (at $2 \sigma$ ). In the hatched region in this domain the "data" are still consistent with $m_{0}=0$ for the inverted ordering, i.e. with an IH spectrum. Hence, if a result within the hatched region is obtained, we can conclude that either $\operatorname{sgn}\left(\Delta m_{31}^{2}\right)>0$ and the spectrum is with partial hierarchy or of the QD type, or $\operatorname{sgn}\left(\Delta m_{31}^{2}\right)<0$ and the spectrum is IH $\left(m_{0} \leq 0.02 \mathrm{eV}\right)$, or QD $\left(m_{0} \gtrsim 0.1 \mathrm{eV}\right)$, or with partial hierarchy $\left(m_{0}^{2} \sim\left|\Delta m_{\mathrm{A}}^{2}\right|\right)$. This situation corresponds, e.g. to the panels of the middle column in Fig. 2, or to the case when the lower branch at $m_{0} \lesssim 0.01 \mathrm{eV}$ in the case of normal ordering (see Fig. (1) can be excluded. Finally, for sufficiently large values of $|\langle m\rangle|^{\text {obs }}$, corresponding to the white regions in Fig. [3, the spectrum is of QD type and no information on $\operatorname{sgn}\left(\Delta m_{31}^{2}\right)$ can be obtained.

Let us add that these results are stable with respect to variations of the oscillation parameters within the present allowed ranges. In particular, they practically do not depend on the value of $\sin ^{2} \theta_{13}$ : no significant changes appear if, instead of the rather large value $\sin ^{2} \theta_{13}=0.03$ adopted in Fig. 3, smaller values are used. As discussed in Section 2, cosmol- 


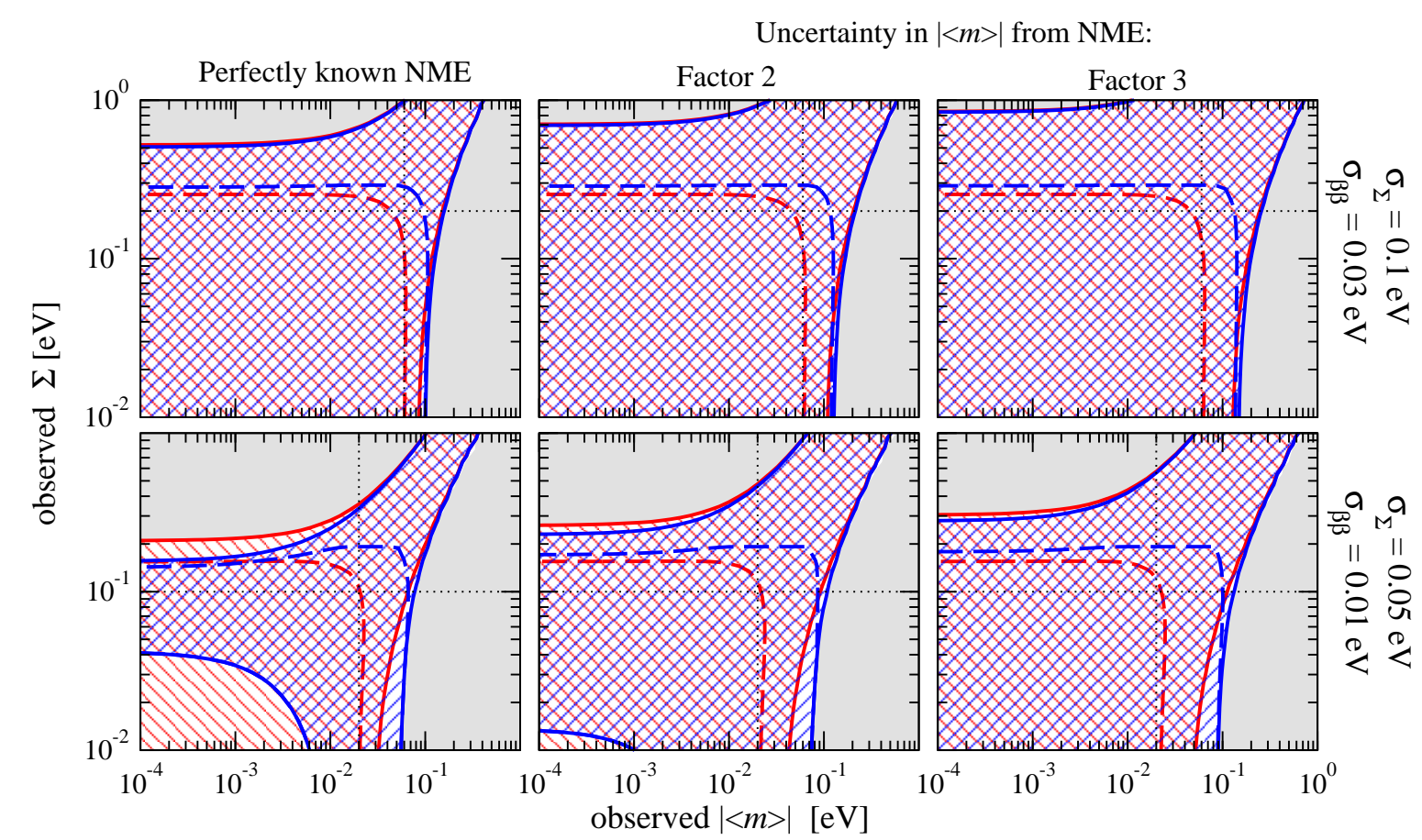

$\begin{array}{ll}\square \square & \text { Normal ordering } \quad--- \text { Normal hierarchical } \square|\langle m\rangle| \text { and } \Sigma \text { inconsistent at } 2 \sigma \\ \mathscr{Z} \text { Inverted ordering } & --- \text { Inverted hierarchical }\end{array}$

$$
\sin ^{2} \theta_{13}=0 \pm 0.002, \sin ^{2} \theta_{12}=0.31 \pm 3 \%, \Delta m_{21}^{2}=8 \times 10^{-5} \pm 2 \%,\left|\Delta m_{31}^{2}\right|=2.2 \times 10^{-3} \pm 3 \%
$$

Figure 4: Information on the neutrino mass spectrum from a combination of $(\beta \beta)_{0 \nu}$-decay data on $|<m>|$ and cosmological data on $\Sigma$. These results are obtained for different assumptions about the errors in the determination of $|\langle m\rangle|$ and $\Sigma$ (rows of panels) and the NME uncertainty factor $F$ (columns of panels). We test whether the $(\beta \beta)_{0 \nu}$-decay and cosmological data are consistent with each other, with normal or inverted mass ordering, with normal ordering and $m_{0}=0$ ( $\mathrm{NH} \nu$-mass spectrum), or with inverted ordering and $m_{0}=0$ (IH spectrum), at $2 \sigma$ C.L. The regions to the right of (above) the vertical (horizontal) dotted lines correspond to non-zero observed $|<m>|^{\text {obs }}\left(\Sigma^{\text {obs }}\right)$ at $2 \sigma$.

ogy provides a sensitive tool to constrain the sum of the neutrino masses $\Sigma \equiv m_{1}+m_{2}+m_{3}$. In the following we investigate what can be learned from data on $(\beta \beta)_{0 \nu}$-decay, combined with information on $\Sigma$ from cosmology. We include the latter by assuming an "observed" value of the sum of the neutrino masses $\Sigma^{\text {obs }}$ with an experimental accuracy $\sigma_{\Sigma}$. Obviously, if $\Sigma^{\text {obs }}>n \sigma_{\Sigma}$, cosmological observations would provide positive evidence for a non-zero $\Sigma^{\text {obs }}$ at the $n \sigma$ C.L.; otherwise an upper bound is obtained. In Fig. 4 we show the results of a combined analysis of a $(\beta \beta)_{0 \nu}$-decay result with information from cosmology as a function of the observed values of $|<m\rangle \mid$ and $\Sigma$ for two sets of representative experimental errors (upper and lower rows) and different assumptions on the uncertainty from the NME. For given values of $|<m>|^{\text {obs }}$ and $\Sigma^{\text {obs }}$ we look for the $\chi^{2}$-minimum for each of the two possible mass orderings. If a minimum is less than 4 , we conclude that the corresponding mass ordering is consistent with the data at $2 \sigma$. These regions are indicated by the hatched areas in Fig. 4. If the $\chi^{2}$-minimum is bigger than 4 for both types of mass orderings, the corresponding values 
of $|<m>|^{\text {obs }}$ and $\Sigma^{\text {obs }}$ are not consistent at $2 \sigma$ within the assumed uncertainties. Such a situation (shown as the shaded regions in Fig. 4) could either result from systematical effects not taken into account in the cosmological data, or can indicate that some mechanism beyond the light Majorana neutrino exchange is operating in $(\beta \beta)_{0 \nu}$-decay [81]. We also test whether the data are consistent with hierarchical spectra, i.e. for each sign of $\Delta m_{31}^{2}$ we test whether $\chi^{2}\left(m_{0}=0\right) \leq 4$. These regions are below the dashed lines in Fig. 4, within the corresponding hatched area. ${ }^{13}$

For experimental errors corresponding to $\sigma_{\beta \beta}=0.03 \mathrm{eV}$ and $\sigma_{\Sigma}=0.1 \mathrm{eV}$ adopted in the upper row of plots in Fig. 4 no distinction between $\operatorname{sgn}\left(\Delta m_{31}^{2}\right)>0$ and $\operatorname{sgn}\left(\Delta m_{31}^{2}\right)<0$ (i.e. normal and inverted ordering) is possible. However, some information can be obtained on whether the spectrum is hierarchical for a given $\operatorname{sgn}\left(\Delta m_{31}^{2}\right)$. In particular, the data from cosmology increase the ability to distinguish between IH and QD spectra in the case of $\operatorname{sgn}\left(\Delta m_{31}^{2}\right)<0$ if $|<m>| \simeq 0.1 \mathrm{eV}$ is observed. This situation corresponds to the case indicated by the hatched region in Fig. 3, where $(\beta \beta)_{0 \nu}$-decay alone can only rule out the normal hierarchical spectrum.

For the more demanding experimental precision of $\sigma_{\beta \beta}=0.01 \mathrm{eV}$ and $\sigma_{\Sigma}=0.05 \mathrm{eV}$, used in the lower row of plots in Fig. 4, a new possibility to distinguish between normal and inverted mass ordering appears. If the $(\beta \beta)_{0 \nu}$-decay data give, e.g. a value of $|<m>|$ in the interval $(0.04-0.07) \mathrm{eV}$ for NME uncertainty factor $F=2$, and the cosmological observations yield an upper bound $\Sigma^{\mathrm{obs}} \leq 2 \sigma_{\Sigma}=0.1 \mathrm{eV}$, the IH spectrum can be established at $2 \sigma$ C.L. This is a qualitatively new method to determine the mass ordering, emerging from a synergy between data from $(\beta \beta)_{0 \nu}$-decay experiments and cosmology. Using $(\beta \beta)_{0 \nu}$-decay data alone it is possible, in principle, to rule out the inverted ordering if $|<m>|^{\text {obs }}<0.01 \mathrm{eV}$ and the error $\sigma_{\beta \beta}$ is sufficiently small. However, as we have shown, the required error is exceedingly small: $\sigma_{\beta \beta} \lesssim$ few $\times 10^{-3} \mathrm{eV}$. In contrast, the conclusion following from a combination of $(\beta \beta)_{0 \nu}$-decay and cosmological data is based on i) the observation of a value of $|<m>|$ compatible with those predicted for the IH spectrum, and ii) an upper bound on $m_{0}$ from cosmological data such that the region where the predictions for $|<m\rangle \mid$ in the cases of normal and inverted hierarchies merge, can be excluded (see Fig. 1). Note that this possibility remains even if a factor 3 uncertainty in the NME is taken into account.

\subsection{Constraining the Majorana CPV Phases}

The possibility of establishing CP-violation due to Majorana phases through the observation of $(\beta \beta)_{0 \nu}$-decay has been studied previously in $[39,41,58]$. In the following we re-consider this problem by applying the $\chi^{2}$-method described in Section 4.2. We discuss first the case of QD spectrum and later consider the possibility of spectrum with inverted hierarchy, $\operatorname{sgn}\left(\Delta m_{\mathrm{A}}^{2}\right)<0$, and $|<m>|^{\text {obs }}$ lying in the IH range of $(0.015-0.05) \mathrm{eV}$. As is clear from eqs. (15) and (20) and the discussion in Section 3 , the dependence of $|<m>|$ on the phase $\alpha_{31}$ is suppressed by the small value of $\sin ^{2} \theta_{13}$. Therefore, we concentrate on the determination of the phase $\alpha_{21}$, while the dependence on $\alpha_{31}$ is taken into account implic-

\footnotetext{
${ }^{13} \mathrm{We}$ adopt the convention to determine the compatibility of the $(\beta \beta)_{0 \nu}$-measurement and cosmological data by evaluating the $\chi^{2}$ for 1 degree-of-freedom. A motivation for this convention is provided by the so-called parameter-goodness-of-fit method discussed in [82]. In the context of that method the single degree-of-freedom corresponds to the one parameter, $m_{0}$, which is comon to $|<m>|$ and $\Sigma$.
} 


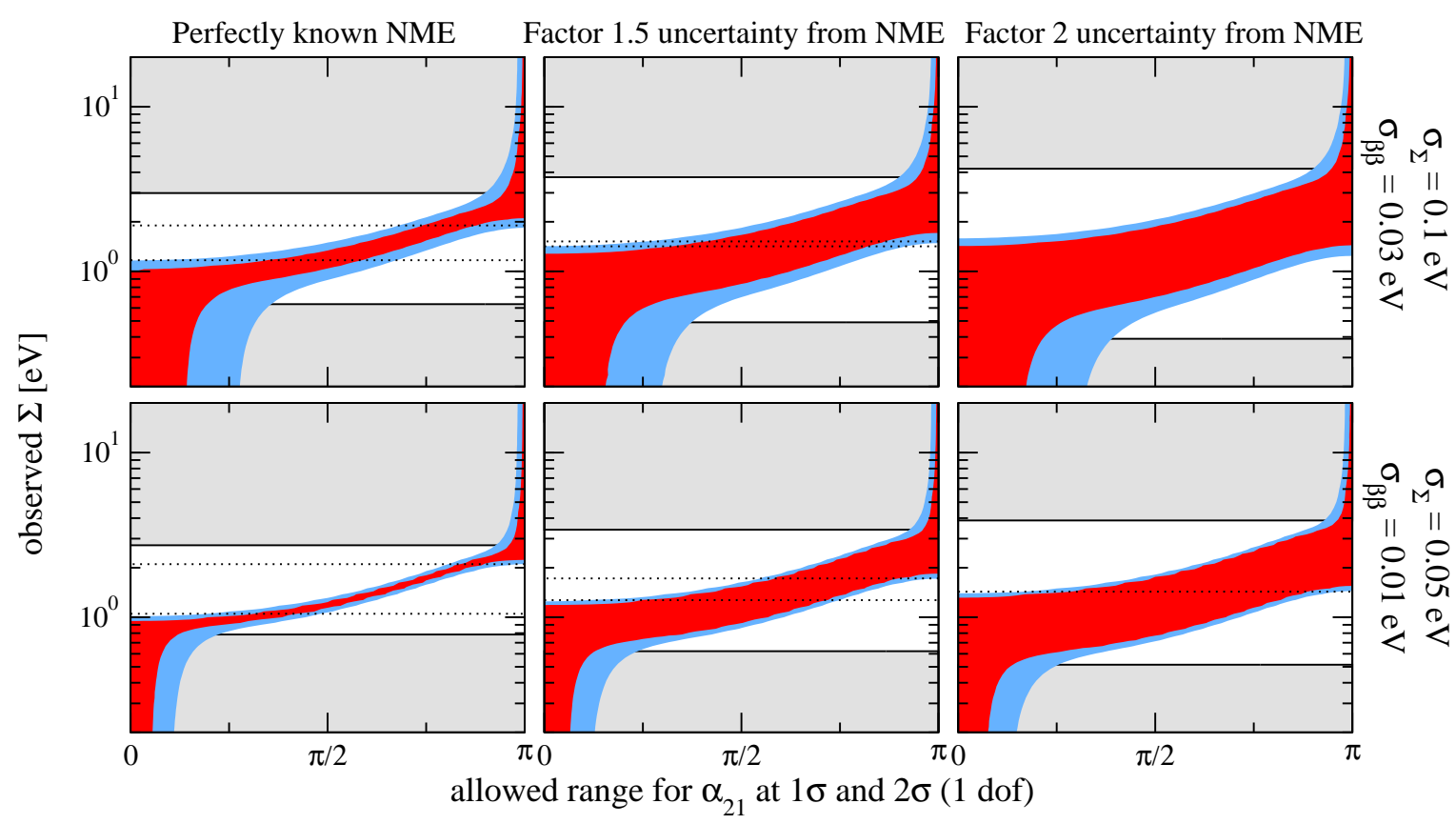

observed $|\langle m\rangle|=0.3 \mathrm{eV} \quad \square \quad|\langle m\rangle|$ and $\Sigma$ inconsistent at $2 \sigma$
$\sin ^{2} \theta_{13}=0 \pm 0.002, \sin ^{2} \theta_{12}=0.31 \pm 3 \%, \Delta m_{21}^{2}=8 \times 10^{-5} \pm 2 \%, \Delta m_{31}^{2}=2.2 \times 10^{-3} \pm 3 \%$

Figure 5: Allowed range for the Majorana phase $\alpha_{21}$ at $1 \sigma$ C.L. (dark-gray/red regions) and $2 \sigma$ C.L. (medium-gray/blue regions) for $|<m>|=0.3 \mathrm{eV}$ as a function of the observed value of $\Sigma$. The shown results are obtained for two sets of assumed errors in the observed $|<m>|$ and $\Sigma$ (rows of panels) and three values of the NME uncertainty factor $F$ (columns of panels). For values of the parameters in the regions between the dotted lines, Majorana $\mathrm{CP}$-violation can be established at $2 \sigma$.

itly by minimising the $\chi^{2}$ with respect to it. For the oscillation parameters we will assume throughout this subsection uncertainties at the few percent level (precise numbers are given in the figures). Such a precision can be reached in upcoming oscillation experiments. In Fig. 5 we show the allowed range for the Majorana phase $\alpha_{21}$ for $|<m>|^{\text {obs }}=0.3 \mathrm{eV}$ as a function of the observed mean value of $\Sigma$. The $1 \sigma(2 \sigma)$ range is obtained by the condition $\Delta \chi^{2}\left(\alpha_{21}\right)=\chi^{2}\left(\alpha_{21}\right)-\chi_{\min }^{2} \leq 1(4)$. Since the allowed range is determined by $\Delta \chi^{2}$ with respect to the $\chi^{2}$-minimum, there is always an "allowed region", irrespectively of whether $\Sigma^{\text {obs }}$ is consistent with the adopted value of $|<m>|^{\text {obs }}$. We indicate in Fig. 5 the region where the "results" of $(\beta \beta)_{0 \nu}$-decay experiment and cosmological observations are inconsistent $\left(\chi_{\min }^{2} \geq 4\right)$ by the light shading. Majorana CP-violation can be established if both $\alpha_{21}=0$ and $\alpha_{21}=\pi$ can be excluded. The relevant regions are indicated by the horizontal dotted lines in Fig. 5. One observes that for $\sigma_{\beta \beta}=0.03 \mathrm{eV}$ and $\sigma_{\Sigma}=0.1 \mathrm{eV}$ used in the upper row of plots, already an uncertainty of a factor of 1.5 in the NME makes it practically impossible to establish CPV. Our results show, in agreement with the results of the previous studies $[41,58]$, that establishing Majorana CP-violation due to $\alpha_{21}$ is very challenging: the errors in the observed $|\langle m\rangle|$ and $\Sigma$ should not exceed approximately $10 \%$ and the NME has to be known within a factor $F \lesssim 1.5$. Although establishing Majorana 


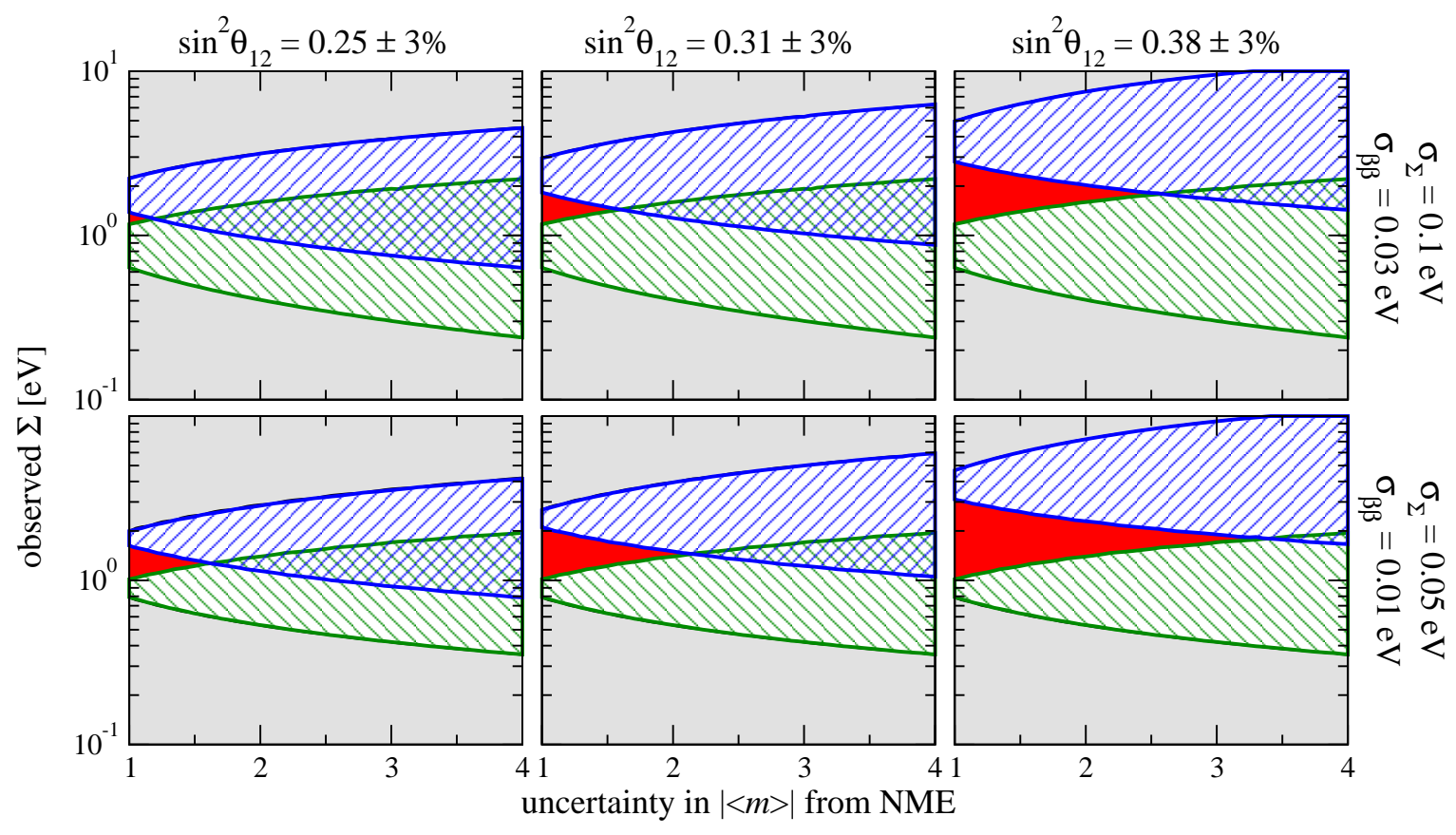

$\begin{array}{ll}\mathscr{Z Z} \text { data consistent with } \alpha_{21}=\pi & \square \text { data consistent with } \alpha_{21}=0 \\ \square|<m>| \text { and } \Sigma \text { inconsistent at } 2 \sigma & \mathrm{CP} \text { violation established at } 2 \sigma\end{array}$

$$
\sin ^{2} \theta_{13}=0 \pm 0.002, \Delta m_{21}^{2}=8 \times 10^{-5} \pm 2 \%, \Delta m_{31}^{2}=2.2 \times 10^{-3} \pm 3 \% \quad \text { observed }|\langle m\rangle|=0.3 \mathrm{eV}
$$

Figure 6: Constraints on the Majorana phase $\alpha_{12}$ at $95 \%$ C.L. from an observed $|<m>|^{\text {obs }}=$ $0.3 \mathrm{eV}$ and (cosmological) "data" on $\Sigma$, as a function of the NME uncertainty factor $F$. Shown are the regions in which i) the "data" are consistent with one of the CP-conserving values $\alpha_{12}=0$ or $\pi$ (hatched), ii) $\Sigma^{\text {obs }}$ is inconsistent with $|<m>|^{\text {obs }}=0.3 \mathrm{eV}$ (light-shaded), and iii) Majorana CP-violation is established (red/dark-shaded). The results are presented for three values of $\sin ^{2} \theta_{\odot}$ within the currently allowed range (columns of panels), and for two choices of the experimental accuracies for $|<m>|$ and $\Sigma$ (rows of panels).

CPV would be a very difficult task, it could be possible to exclude a certain fraction of the full parameter space of the phase $\alpha_{21}{ }^{14}$ by using the data on $|<m>|$ and $\Sigma$. In particular, in many cases it could be possible to exclude one of the CP-conserving values of $\alpha_{21}$, $\alpha_{21}=0$ or $\alpha_{21}=\pi$, corresponding to specific relative CP-parities of the neutrinos $\nu_{1}$ and $\nu_{2}$. The sensitivity to $\alpha_{21}$ depends significantly on the value of the mixing angle $\theta_{\odot}[41,58]$. As is discussed in Sec. 3 (see eq. (22) ), for fixed $m_{0}$ the allowed range of $|<m\rangle \mid$ is given by $m_{0} \cos 2 \theta_{\odot} \leq|<m>| \leq m_{0}$. Therefore, the allowed range increases for smaller values of $\cos 2 \theta_{\odot}$, which makes it easier to exclude the extreme values of $|<m>|$, corresponding to the CP-conserving configurations. This effect is clearly shown in Fig. 6, where the three columns of panels correspond to different values of $\sin ^{2} \theta_{\odot}$. We use the current best fit point as well as values between the present $2 \sigma$ and $3 \sigma$ limits. In Fig. [6 we assume an observation

\footnotetext{
${ }^{14}$ This situation is similar to the case of the determination of the Dirac CP phase $\delta$ by long-baseline oscillation experiments, see [83] for a recent discussion and a list of references.
} 
of $|<m>|^{\text {obs }}=0.3 \mathrm{eV}$, which implies a QD spectrum. Adopting representative values for the experimental accuracies on $|<m>|$ and $\Sigma$, and scanning values of $\Sigma^{\text {obs }}$ and the uncertainty in the NME, we test first whether $\Sigma^{\text {obs }}$ and $|<m>|^{\text {obs }}$ are consistent at $2 \sigma$ C.L. $\left(\chi_{\min }^{2} \leq 4\right)$. If they are, we test whether the phase $\alpha_{21}$ is consistent with the CP-conserving values 0 and $\pi\left(\chi^{2}\left(\alpha_{21}=0, \pi\right) \leq 4\right)$. These two cases are marked by the hatched regions in Fig. 6. The double-hatched areas, where the regions for $\alpha_{21}=0$ and $\pi$ overlap, correspond to the worst situation, where no information on $\alpha_{21}$ can be obtained and the full range $[0, \pi]$ is allowed by the data. If the values of $\Sigma^{\text {obs }}$ and $|<m>|^{\text {obs }}$ are consistent and both CP-conserving solutions for $\alpha_{21}$ can be excluded, Majorana CP-violation can be established at 95\% C.L., and we indicate the corresponding regions in red/dark-shading. Clearly, establishing Majorana CP-violation becomes possible only under rather specific conditions. For $\sin ^{2} \theta_{\odot} \gtrsim 0.31$ and $\sim 10 \%$ errors in the measured $|<m>|^{\text {obs }}$ and $\Sigma^{\text {obs }}$ (upper middle and right panels in Fig. (6)), the NME has to been known to better than within a factor of 1.5. For smaller values of the errors, $\sigma_{\beta \beta} \sim 0.01 \mathrm{eV}$ and $\sigma_{\Sigma} \sim 0.05 \mathrm{eV}$, Majorana CP-violation could be established even for $F \cong 2$ (lower middle and right panels). If, however, $\sin ^{2} \theta_{\odot} \cong 0.25$, the NME uncertainty has to be small, $F \leq 1.5$ and the indicated high precision in the measurement of $|<m>|^{\text {obs }}$ and $\Sigma^{\text {obs }}$ has to be achieved. Finally, the Majorana phase $\alpha_{21}$ has to have a value approximately in the interval $\sim(\pi / 4-3 \pi / 4)$.

Consider next the possibility to establish Majorana CP-violation assuming that the $\nu$ mass spectrum is known to be with inverted ordering, $\operatorname{sgn}\left(\Delta m_{\mathrm{A}}^{2}\right)<0$, and that the observed value of $|\langle m\rangle|$ lies in the IH region of a few $\times 10^{-2} \mathrm{eV}$. Knowing that this would require a rather precise measurement of $|\langle m\rangle|$, we use for the experimental error on $|\langle m\rangle|$ the value $\sigma_{\beta \beta}=4 \times 10^{-3} \mathrm{eV}$. For the sum of neutrino masses $\Sigma$ we adopt the error $\sigma_{\Sigma}=4 \times 10^{-2} \mathrm{eV}$. In Fig. [7] we show the sensitivity to Majorana CP-violation for three representative mean values of $|\langle m\rangle|$ from the IH region, $|<m>|^{\text {obs }}=0.018 ; 0.032 ; 0.047 \mathrm{eV}$, and two mean values of $\sin ^{2} \theta_{\odot}, \sin ^{2} \theta_{\odot}=0.31 ; 0.38$. The allowed regions in all panels of this figure are bounded from below by a straight line at $\Sigma^{\text {obs }}=0.014 \mathrm{eV}$ : below this value $\Sigma$ becomes inconsistent with the adopted value of $\left|\Delta m_{\mathrm{A}}^{2}\right|$.

The upper row of panels corresponds to the present best fit point of $\sin ^{2} \theta_{\odot}$. For $|<m>|^{\text {obs }}=0.018 \mathrm{eV}$ (left panel), the $2 \sigma$ interval of allowed values of $|<m>|$ always includes the minimal value of $|\langle m\rangle|$ for the IH spectrum (see eq. (18)): $|\langle m\rangle|^{\min \cong}$ $\sqrt{\left|\Delta m_{A}^{2}\right|} \cos 2 \theta_{\odot}$. The latter corresponds to the CP-conserving value $\alpha_{21}=\pi$. Thus, for the chosen values of $|\langle m\rangle|^{\text {obs }}, \sigma_{\beta \beta}, \sigma_{\Sigma}$ and $\sin ^{2} \theta_{\odot}$, it is impossible to establish Majorana CP-violation. However, if $F \leq 3$, it will be possible to conclude that $\alpha_{21}$ has a nonzero value, $\alpha_{21} \neq 0$. Thus, if $\mathrm{CP}$ is conserved, the neutrinos $\nu_{1}$ and $\nu_{2}$ cannot have the same CP-parities.

The value $|<m>|^{\text {obs }}=0.032 \mathrm{eV}$ adopted in the middle panel corresponds to the case when $|<m>|$ (with the experimental uncertainty included) satisfies $|<m>|^{\text {min }}<|<m>|<$ $|<m>|^{\max },|<m>|^{\max }$ being maximal value of $|<m>|$ predicted in the case of IH spectrum

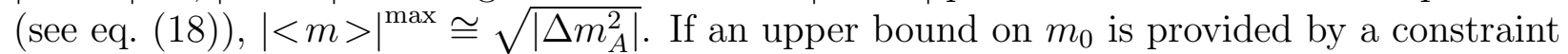
on $\Sigma$, Majorana CP-violation can be established, as evident from the red/dark-shaded region in the panel. If the observed value of $\Sigma$ becomes too large, the "data" becomes consistent with the "upturn" of the IH-branch (see Fig. 1), which implies that the CP-conserving value $\alpha_{21}=\pi$ is allowed. For $\Sigma^{\text {obs }} \lesssim 0.2 \mathrm{eV}$ and uncertainties in the NME $F \gtrsim 1.5-2$, the "data" become consistent with $|<m>|^{\max }$, i.e. with $\alpha_{21}=0$. 


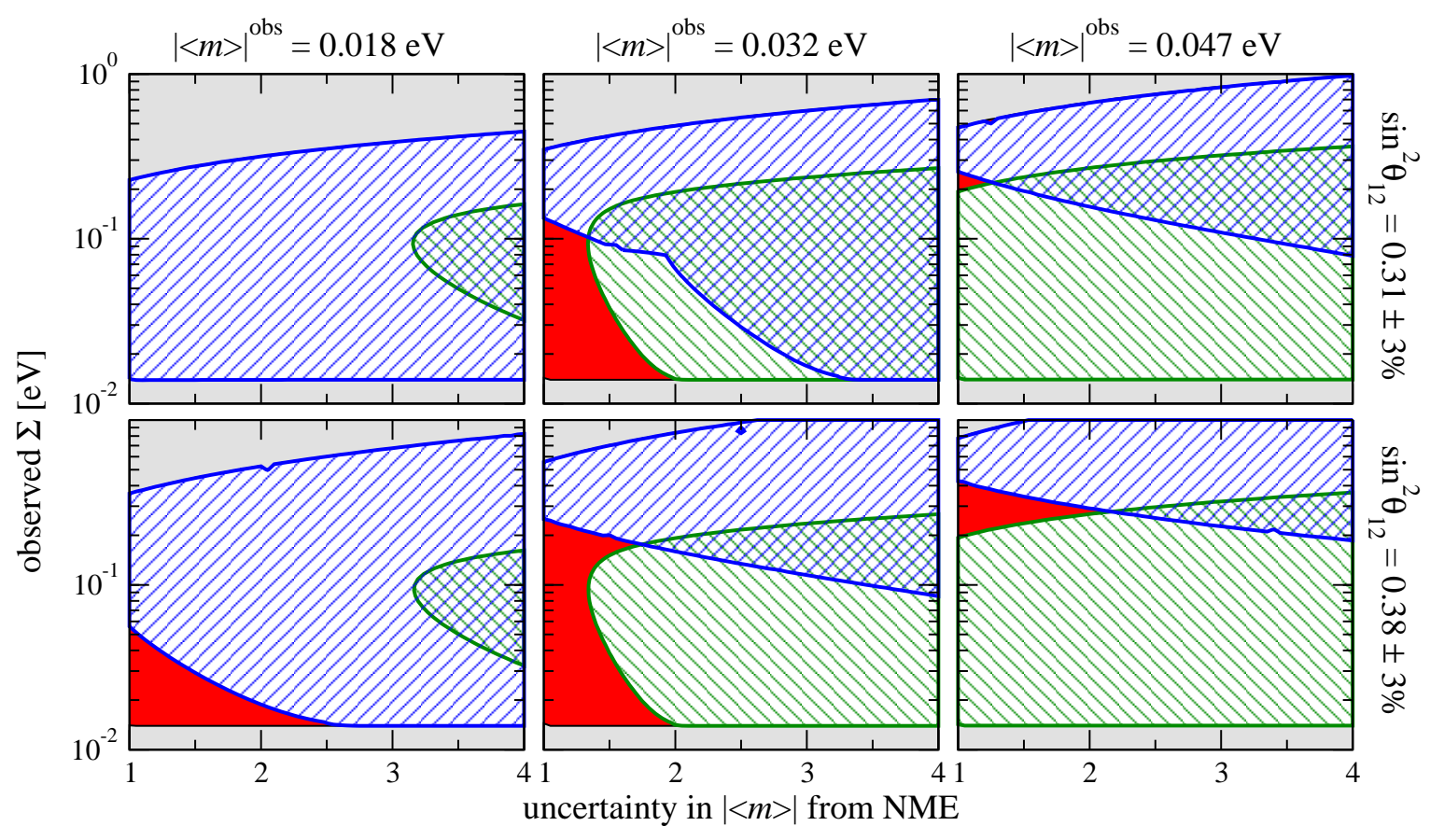

$$
\begin{array}{cc}
\square 2 D \text { data consistent with } \alpha_{21}=\pi & \square \text { data consistent with } \alpha_{21}=0 \\
\square|<m>| \text { and } \Sigma \text { inconsistent at } 2 \sigma & \text { CP violation established at } 2 \sigma \\
\sin ^{2} \theta_{13}=0 \pm 0.002, \Delta m_{21}^{2}=8 \times 10^{-5} \pm 2 \%, \Delta m_{31}^{2}=-2.2 \times 10^{-3} \pm 3 \%, \quad \sigma_{\beta \beta}=0.004 \mathrm{eV}, \sigma_{\Sigma}=0.04 \mathrm{eV}
\end{array}
$$

Figure 7: Constraints on the Majorana phase $\alpha_{12}$ at 95\% C.L. for the inverted mass ordering from observed values $|\langle m\rangle|^{\text {obs }}=0.018,0.032,0.047 \mathrm{eV}$ and (cosmological) "data" on $\Sigma$, as a function of the NME uncertainty factor $F$. Shown are the regions in which i) the "data" are consistent with one of the CP-conserving values $\alpha_{12}=0$ or $\pi$ (hatched), ii) $\Sigma^{\text {obs }}$ is inconsistent with $|<m>|^{\text {obs }}$ (light-shaded), and iii) Majorana CP-violation is established (red/dark-shaded). The upper (lower) row of panels corresponds to $\sin ^{2} \theta_{\odot}=0.31 ;(0.38)$.

For the third representative value of $|<m>|^{\text {obs }}=0.047 \mathrm{eV}$ (right panels), $|<m>|^{\text {max }}$ lies in the $2 \sigma$ interval of allowed values of $|<m\rangle \mid$. This implies that Majorana CP-violation can only be established if the "data" on $\Sigma$ constrains $m_{0}$ precisely in the range corresponding to a neutrino mass spectrum with partial inverted hierarchy, such that neither $|\langle m\rangle|^{\max }$ (i.e. the horizontal branch at $\alpha_{21}=0$ ), nor the upturn of the lower (minimal) branch at $\alpha_{21}=\pi$, are compatible with the "data". As can be seen in Fig. [7 this is marginally possible for $\sin ^{2} \theta_{\odot}=0.31$ (upper row), but some window exists for $\sin ^{2} \theta_{\odot}=0.38$ (lower row) if the NME uncertainty factor $F \leq 2$.

\section{Conclusions}

In the present article we have reanalysed the potential contribution of future $(\beta \beta)_{0 \nu^{-}}$ decay experiments to the studies of neutrino mixing. We have considered $3-\nu$ mixing and as- 
sumed massive Majorana neutrinos and $(\beta \beta)_{0 \nu}$-decay generated only by the $(V-A)$ charged current weak interaction via the exchange of the three Majorana neutrinos. In this framework we investigated which information can be obtained from a measurement of the effective Majorana mass $|<m>|$ i) on the type of neutrino mass spectrum (NH, IH, QD, etc.) ii) on the absolute scale of neutrino masses, and iii) on the Majorana CP-violating phases. As input in the analysis we used the results of recent studies of the prospective precision that can be achieved in the future measurements of neutrino oscillation parameters on which $|<m>|$ depends. We performed a $\chi^{2}$ analysis taking into account experimental and theoretical errors, as well as the uncertainty implied by the imprecise knowledge of the corresponding nuclear matrix element (NME).

We show how the possibility to discriminate between the NH, IH and QD spectra depends on the mean value and the experimental error of $|\langle m\rangle|$, and on the NME uncertainty. Furthermore, we combine the information on $|<m>|$ from a $(\beta \beta)_{0 \nu}$-decay experiment, with a constraint on the sum of the neutrino masses, $\Sigma$, which can be obtained from cosmological observations. In this case, we investigate the role of the accuracies on $|\langle m\rangle|$ and $\Sigma$, as well as on the NME uncertainty, in determining the type of neutrino mass spectrum. The constraints on Majorana CP-violation phases in the neutrino mixing matrix, that can be obtained from a measurement of $|\langle m\rangle|$ and $\Sigma$ in the cases when i) the observed $|\langle m\rangle| \sim$ few $\times 10^{-1} \mathrm{eV}$ (QD spectrum), and ii) $\operatorname{sgn}\left(\Delta m_{\mathrm{A}}^{2}\right)<0$ and the observed $|<m>| \sim$ few $\times$ $10^{-2} \mathrm{eV}$, are also analyzed in detail. We have estimated the required experimental accuracies on both types of measurements, and the required precision in the NME permitting to address the issue of Majorana CP-violation in the lepton sector.

Our results show that, in general, getting quantitative information on the neutrino mass and mixing parameters from a measurement of the $(\beta \beta)_{0 \nu}$-decay half-life is rather insensitive to the errors on the input neutrino oscillation parameters as long as the errors are smaller than $\sim 10 \%$. However, constraints on the absolute neutrino mass scale, on the type of neutrino mass spectrum and on the Majorana CP-violation phase one can obtain depend critically on the measured mean value of $|\langle m\rangle|$ (and $\Sigma$ ), on the precision reached in the measurement of $|\langle m\rangle|$ (and $\Sigma$ ), and on the uncertainty in the knowledge of the value of the relevant $(\beta \beta)_{0 \nu}$-decay nuclear matrix element. The most challenging of these physics goals is obtaining quantitative information on Majorana CP-violation phases. The sensitivity to the latter depends crucially also on the value of $\sin ^{2} \theta_{\odot}$. Establishing Majorana CPviolation using data on $|\langle m\rangle|$ and $\Sigma$ in the case of QD spectrum, for instance, would require for $\sin ^{2} \theta_{\odot} \cong 0.31, \mathrm{a} \sim 10 \%$ (or smaller) errors in the measured $|<m>|^{\text {obs }}$ and $\Sigma^{\text {obs }}$ and knowledge of the relevant NME with an uncertainty corresponding to a factor $F \leq 1.5$. For smaller values of the errors and/or larger values of $\sin ^{2} \theta_{\odot}$, say $\sin ^{2} \theta_{\odot} \cong 0.38$, it could be possible to obtain evidence of Majorana CP-violation at $2 \sigma$ C.L. even for $F \cong 2$. If, however, $\sin ^{2} \theta_{\odot} \cong 0.25$, exceedingly high precision in the measurements of $|<m>|$ and $\Sigma$ and NME uncertainty smaller than 1.5 is required. In all these cases the Majorana phase $\alpha_{21}$ has to have also a value approximately in the interval $\sim(\pi / 4-3 \pi / 4)$.

Future $(\beta \beta)_{0 \nu}$-decay experiments have a remarkable physics potential. They can establish the Majorana nature of neutrinos with definite mass $\nu_{j}$. If the latter are Majorana particles, the $(\beta \beta)_{0 \nu}$-decay experiments can provide constraints on the absolute scale of neutrino masses and on the type of neutrino mass spectrum. They can also provide unique information on the Majorana CP-violation phases present in the PMNS neutrino mixing matrix. The 
measurement of $|\langle m\rangle|$ (and $\Sigma$ ) with sufficiently small error and sufficiently precise knowledge of the values of the relevant $(\beta \beta)_{0 \nu}$-decay nuclear matrix elements $(F<2)$ is crucial for obtaining significant quantitative information on the neutrino mass and mixing parameters from a measurement of $(\beta \beta)_{0 \nu}$-decay half-life. The remarkably challenging physics goal of getting evidence for Majorana CP-violation in the lepton sector could possibly be achieved only if in addition $\sin ^{2} \theta_{\odot}$ and the Majorana CP-violation phase $\alpha_{21}$ have favorable values.

\section{Acknowledgments}

We would like to thank S. M. Bilenky for useful discussions. S.P. would like to thank the Elementary Particle Physics Sector at SISSA for kind hospitality during the first stage of this study. This work was supported in part by the Italian MIUR and INFN under the programs on "Fisica Astroparticellare" (S.T.P.). The work of T.S. is supported by a "Marie Curie Intra-European Fellowship within the 6th European Community Framework Programme."

\section{References}

[1] B. T. Cleveland et al., Astrophys. J. 496 (1998) 505; Y. Fukuda et al. [Kamiokande Collaboration], Phys. Rev. Lett. 77 (1996) 1683; J. N. Abdurashitov et al. [SAGE Collaboration], J. Exp. Theor. Phys. 95 (2002) 181; T. Kirsten et al. [GALLEX and GNO Collaborations], Nucl. Phys. B (Proc. Suppl.) 118 (2003) 33; C. Cattadori, Talk given at $\nu$ '04 International Conference, June 14-19, 2004, Paris, France.

[2] S. Fukuda et al. [Super-Kamiokande Collaboration], Phys. Lett. B539 (2002) 179.

[3] Q. R. Ahmad et al. [SNO Collaboration], Phys. Rev. Lett. 87 (2001) 071301; ibid. 89 (2002) 011301 and 011302; S. N. Ahmed et al., Phys. Rev. Lett. 92 (2004) 181301; B. Aharmim et al., nucl-ex/0502021.

[4] Y. Fukuda et al. [Super-Kamiokande Collaboration], Phys. Rev. Lett. 81 (1998) 1562.

[5] E. Kearns [Super-Kamiokande Collaboration], Talk given at $\nu$ '04 International Conference, June 14-19, 2004, Paris, France (http://neutrino2004.in2p3.fr).

[6] K. Eguchi et al. [KamLAND Collaboration], Phys. Rev. Lett. 90 (2003) 021802.

[7] M. H. Ahn et al. [K2K Collaboration], Phys. Rev. Lett. 90 (2003) 041801.

[8] C. Athanassopoulos et al. [LSND Collaboration], Phys. Rev. Lett. 81 (1998) 1774.

[9] Y. Ashie et al. [Super-Kamiokande Collaboration], Phys. Rev. Lett. 93 (2004) 101801.

[10] T. Araki et al. [KamLAND Collaboration], hep-ex/0406035.

[11] E. Aliu et al. [K2K Collaboration], hep-ex/0411038.

[12] V. Gribov and B. Pontecorvo, Phys. Lett. B28 (1969) 493. 
[13] B. Pontecorvo, Zh. Eksp. Teor. Fiz. (JETP) 33 (1957) 549; ibid. 34 (1958) 247; ibid. 53 (1967) 1717; Z. Maki, M. Nakagawa and S. Sakata, Prog. Theor. Phys. 28 (1962) 870.

[14] M. Maltoni et al., New J. Phys. 6122 (2004) 122.

[15] M. Sorel, J. Conrad and M. Shaevitz, Phys. Rev. D 70 (2004) 073004.

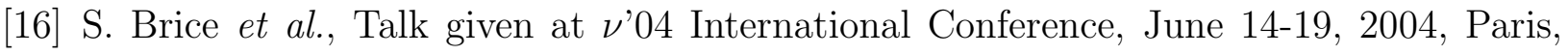
France (http://neutrino2004.in2p3.fr).

[17] S.M. Bilenky, J. Hosek and S.T. Petcov, Phys. Lett. B94 (1980) 495.

[18] J. Schechter and J.W.F. Valle, Phys. Rev. D22 (1980) 2227; M. Doi et al., Phys. Lett. B102 (1981) 323; J. Bernabeu and P. Pascual, Nucl. Phys. B 228 (1983) 21.

[19] S.M. Bilenky, S. Pascoli and S.T. Petcov, Phys. Rev. D64 (2001) 053010.

[20] M. Apollonio et al., Phys. Lett. B466 (1999) 415; F. Boehm et al., Phys. Rev. Lett. 84 (2000) 3764.

[21] S.T. Petcov, Nucl. Phys. B (Proc. Suppl.) 143 (2005) 159 (hep-ph/0412410).

[22] A. Bandyopadhyay et al., Phys. Lett. B608 (2005) 115.

[23] J.N. Bahcall, M.C. Gonzalez-Garcia and C. Peña-Garay, hep-ph/0406294.

[24] S.M. Bilenky et al., Phys. Rev. D56 (1996) 4432.

[25] S. Pascoli and S.T. Petcov, Phys. Lett. B544 (2002) 239; ibid. B580 (2004) 280.

[26] P. Huber et al., Phys. Rev. D70 (2004) 073014; Nucl. Phys. B (Proc. Suppl.) 145 (2005) 190 (hep-ph/0412133).

[27] V. Martemyanov et al., Phys. Atom. Nucl. 66, 1934 (2003); H. Minakata et al., Phys. Rev. D 68, 033017 (2003) [Erratum-ibid. D 70, 059901 (2004)]; P. Huber et al., Nucl. Phys. B 665 (2003) 487; see also: K. Anderson et al., hep-ex/0402041.

[28] A. Bandyopadhyay et al., Phys. Lett. B B581 (2004) 62.

[29] S. Choubey and S.T. Petcov, Phys. Lett B594 (2004) 333.

[30] A. Bandyopadhyay et al., Phys. Rev. D 72 (2005) 033013.

[31] M. Freund et al., Nucl. Phys. B578 (2000) 27; C. Albright et al., physics/0411123.

[32] P. Huber, M. Lindner and W. Winter, Nucl. Phys. B 654 (2003) 3; H. Minakata, H. Nunokawa and S. J. Parke, Phys. Rev. D 68 (2003) 013010.

[33] O. Mena Requejo, S. Palomares-Ruiz and S. Pascoli, Phys. Rev. D 72 (2005) 053002.

[34] J. Bernabéu, S. Palomares-Ruiz and S. T. Petcov, Nucl. Phys. B 669 (2003) 255; S. Palomares-Ruiz and S.T. Petcov, Nucl. Phys. B 712 (2005) 392. 
[35] P. Huber, M. Maltoni and T. Schwetz, Phys. Rev. D 71 (2005) 053006.

[36] P. Langacker et al., Nucl. Phys. B282 (1987) 589.

[37] S. Pascoli, S.T. Petcov and C.E. Yaguna, Phys. Lett. B564 (2003) 241.

[38] M. Fukugita and T. Yanagida, Phys. Lett. B174 (1986) 45.

[39] V. Barger et al., Phys. Lett. B540 (2002) 247.

[40] A. de Gouvea, B. Kayser and R. Mohapatra, Phys. Rev. D D67 (2003) 053004.

[41] S. Pascoli, S.T. Petcov and W. Rodejohann, Phys. Lett. B549 (2002) 177.

[42] S.M. Bilenky and S.T. Petcov, Rev. Mod. Phys. 59 (1987) 671.

[43] S.T. Petcov, Phys. Lett. B110 (1982) 245.

[44] P.H. Frampton, S.T. Petcov and W. Rodejohann, Nucl. Phys. B 687 (2004) 31; S.T. Petcov and W. Rodejohann, Phys. Rev. D D71 (2005) 073002, and references quoted therein.

[45] P. Minkowski, Phys. Lett. B67 (1977) 421; M. Gell-Mann, P. Ramond, and R. Slansky in Supergravity, p. 315, edited by F. Nieuwenhuizen and D. Friedman, North Holland, Amsterdam, 1979; T. Yanagida, Proc. of the Workshop on Unified Theories and the Baryon Number of the Universe, edited by O. Sawada and A. Sugamoto, KEK, Japan 1979; R.N. Mohapatra, G. Senjanovic, Phys. Rev. Lett. 44 (1980) 912.

[46] A. Atre, V. Barger and T. Han, hep-ph/0502163.

[47] A. Morales and J. Morales, Nucl. Phys. Proc. Suppl. 114 (2003) 141.

[48] C. Aalseth et al., hep-ph/0412300.

[49] S.R. Elliot and P. Vogel, Annu. Rev. Nucl. Part. Sci. 52 (2002) 115.

[50] L. Wolfenstein, Phys. Lett. B107 (1981) 77; S.M. Bilenky, N.P. Nedelcheva and S.T. Petcov, Nucl. Phys. B247 (1984) 61; B. Kayser, Phys. Rev. D30 (1984) 1023.

[51] H.V. Klapdor-Kleingrothaus et al., Nucl. Phys. Proc. Suppl. 100 (2001) 309.

[52] C.E. Aalseth, F.T. Avignone et al., Phys. of Atomic Nuclei 63 (2000) 1225.

[53] H.V. Klapdor-Kleingrothaus et al., Phys. Lett. B586 (2004) 198.

[54] A. Barabash et al., JETP Lett. 80 (2004) 377.

[55] S. Capelli et al. [CUORICINO Collaboration], hep-ex/0505045.

[56] J. Schechter and J.W.F. Valle, Phys. Rev. D25 (1982) 2951; E. Takasugi, Phys. Lett. B 149 (1984) 372. 
[57] S.T. Petcov, New J. Phys. 6 (2004) 109 (http://stacks.iop.org/1367-2630/6/109); Talk given at the Nobel Symposium (N 129) on Neutrino Physics, August 19 - 24, 2004, Haga Slot, Enköping, Sweden, hep-ph/0504110; S. Pascoli and S.T. Petcov, hep-ph/0308034.

[58] S. Pascoli, S.T. Petcov and L. Wolfenstein, Phys. Lett. B524 (2002) 319; S. Pascoli and S.T. Petcov, hep-ph/0111203.

[59] S. Pascoli, S.T. Petcov and W. Rodejohann, Phys. Lett. B558 (2003) 141.

[60] S.M. Bilenky et al., Phys. Lett. B465 (1999) 193; V. Barger and K. Whisnant, Phys. Lett. B456 (1999) 194; F. Vissani, JHEP 06 (1999) 022; M. Czakon et al., hep-ph/0003161; H.V. Klapdor-Kleingrothaus, H. Päs and A.Yu. Smirnov, Phys. Rev. D63 (2001) 073005.

[61] W. Rodejohann, Nucl. Phys. B597 (2001) 110 and hep-ph/0203214, K. Matsuda et al., Phys. Rev. D63 (2001) 077301; T. Fukuyama et al., Phys. Rev. D64 (2001) 013001; F. Deppisch, H. Päs and J. Suhonen, hep-ph/0409306; A. Joniec and M. Zralek, hep-ph/0411070.

[62] H. Murayama and C. Peña-Garay, Phys. Rev. D69 (2004) 031301.

[63] G. L. Fogli et al., Phys. Rev. D 70 (2004) 113003; A. Strumia and F. Vissani, hep-ph/0503246

[64] J. Beacom and M. Vagins, Phys. Rev. Lett. 93, 171101 (2004).

[65] A. Bandyopadhyay et al., Phys. Rev. D67 (2003) 113011; H. Minakata et al., hep-ph/0407326.

[66] S.T. Petcov and A.Yu. Smirnov, Phys. Lett. B322 (1994) 109.

[67] A. Bandyopadhyay et al., Phys. Lett. B583 (2004) 134.

[68] S.T. Petcov, Phys. Lett. B214 (1988) 259.

[69] Y. Itow et al., "The JHF-Kamioka neutrino project," hep-ex/0106019.

[70] D. S. Ayres et al. [NOvA Collaboration], hep-ex/0503053.

[71] F. Perrin, Comptes Rendus 197 (1933) 868; E. Fermi, Nuovo Cim. 11 (1934) 1.

[72] V. Lobashev et al., Nucl. Phys. B (Proc. Suppl.) 91 (2001) 280.

[73] C. Weinheimer et al., Nucl. Phys. Proc. Suppl. 118 (2003) 279.

[74] D.N. Spergel et al. [WMAP Collaboration], Astrophys. J. Suppl. 148 (2003) 175.

[75] S. Hannestad, astro-ph/0303076 O. Elgaroy and O. Lahav, astro-ph/0303089.

[76] W. Hu and M. Tegmark, Astrophys. J. Lett. 514 (1999) 65; M. Tegmark, hep-ph/0503257; J. Lesgourgues, S. Pastor and L. Perotto, Phys. Rev. D70 (2004) 045016. 
[77] M. Kaplinghat, L. Knox and Y. S. Song, Phys. Rev. Lett. 91 (2003) 241301.

[78] V. A. Rodin et al., Phys. Rev. C68 (2003) 044302 and nucl-th/0503063.

[79] S.M. Bilenky and S.T. Petcov, hep-ph/0405237.

[80] M. Ciuchini et al., JHEP 0107 (2001) 013.

[81] K.S. Babu and R.N. Mohapatra, Phys. Rev. Lett. 75 (1995) 2276; M. Hirsch, H.V. Klapdor-Kleingrothaus and S. G. Kovalenko, Phys. Rev. D 53 (1996) 1329; M. Hirsch, H.V. Klapdor-Kleingrothaus and O. Panella, Phys. Lett. B 374 (1996) 7.

[82] M. Maltoni and T. Schwetz, Phys. Rev. D 68 (2003) 033020.

[83] P. Huber, M. Lindner and W. Winter, JHEP 0505 (2005) 020. 